\title{
Distribution Network Optimization with Scattered Generator Integration Using Immune-Clonal Selection Method
}

\author{
Ramadoni Syahputra ${ }^{1 *}$, Indah Soesanti ${ }^{2}$ \\ ${ }^{1}$ Department of Electrical Engineering, Faculty of Engineering, Universitas Muhammadiyah Yogyakarta, Jl. Brawijaya Tamantirto \\ Kasihan, Yogyakarta 55183, Indonesia \\ ${ }^{2}$ Department of Electrical Engineering and Information Technology, Faculty of Engineering, Universitas Gadjah Mada, Jl. Grafika 2, \\ Kampus UGM, Yogyakarta 55281, Indonesia
}

\section{Abstract}

This paper proposes distribution network optimization with scattered generator integration using the immune-clonal selection (ICS) method. Nowadays, the high popularity of scattered generators (SG) has made distribution networks essential to manage appropriately. This interest is because SG is usually injected into the distribution network due to the ease of accessing the network and the voltage level of the distribution network, which is easier for SG to reach. However, the presence of SG as a distribution network is increasingly dynamic, so that appropriate techniques are needed to achieve adequate network performance through network optimization. The ICS method is expected to be the right solution for this task. The ICS technique was chosen for its excellence in accurately optimizing for multi-objectives while avoiding premature convergence to local minima. The ICS approach was applied to IEEE model distribution networks of 33-bus and 71-bus. The optimization results show that the effectiveness and superiority of the ICS method, which is indicated by shallow power losses with a better voltage profile, and the load balance on each feeder is maintained.

\section{Keywords:}

Distribution Network;

Immune-clonal Selection;

Multi-objective Optimization;

Reconfiguration;

Scattered Generators.

\section{Article History:}

Received: 21 August 2021

Revised: $\quad 30$ October 2021

Accepted: $05 \quad$ November 2021

Published: $01 \quad$ December 2021

\section{1- Introduction}

Scattered generators (SG) are characterized as small-scale electric power generators. SG has become synonymous with renewable energy sources with less than $10 \mathrm{MW}$ capacity during the last two decades of power production technology development [1]. Since renewable energy sources are often positioned following their potential energy sources, the SG's location is unpredictable, allowing it to be connected to the distribution or transmission networks at random locations [2-4]. Renewable energy sources which are currently widely applied in the world are solar energy [5, $6]$ and wind [7]. However, micro-hydropower plants $[8,9]$ are still in great demand to utilize the available waterways. The application of renewable energy is becoming increasingly popular because the supply of fossil fuels is becoming increasingly scarce, and environmental problems are becoming more severe [10]. In this regard, the Indonesian government is strongly dedicated to stimulating the construction of renewable energy power plants, specifically by setting a target of renewable energy contributing $23 \%$ of the country's electricity supply by 2025 [11]. Scattered renewable energy generators connected into the interconnection network play a critical role in assisting the government program [12-14].

\footnotetext{
*CONTACT: ramadoni@umy.ac.id
}

DOI: http://dx.doi.org/10.28991/esj-2021-01312

(C) 2021 by the authors. Licensee ESJ, Italy. This is an open access article under the terms and conditions of the Creative Commons Attribution (CC-BY) license (https://creativecommons.org/licenses/by/4.0/). 
The integration of SG into the electric power system increased the voltage on distribution networks, particularly in locations near the SG source $[15,16]$. Voltage growth should be regulated to avoid an excessive increase in voltage [17]. Traditionally, this voltage is controlled by the distribution network operator. The development of increasingly advanced technology in the operation of electric power systems, especially distribution networks, means the use of information and communication technology (ICT) is unavoidable. ICT applications in distribution network operations are very helpful in improving distribution network performance [18]. Neat planning, speed of response, and measurable control are essential factors present with the presence of ICT in the power distribution system. One of the critical roles of ICT in the distribution network is the distribution network reconfiguration operation to reduce power loss and improve the voltage profile in the distribution system $[19,20]$.

The distribution network is the component of the electric power system with the highest power losses compared to the electric power transmission network. Good distribution network management is an imperative key to the successful operation of the electric power system [21]. Generally, the distribution network is operated in a radial network topology [22], although the network is very complex and has many feeders. The advantages of radial networks are simplicity and low cost of investment and operation. However, the radial network has low reliability because it is highly dependent on a distribution network feeder. However, the current distribution network has been designed with connections through switches spread over many places in the distribution network. This condition is advantageous because network reconfiguration is the simplest but most effective way to improve distribution network performance. Network reconfiguration is relatively more profitable than other techniques, such as installing capacitors, voltage regulators, or changing the system voltage to a higher one [23].

The reconfiguration of distribution networks is a complicated, combinatorial, and multi-objective problem. In this example, there are numerous imperfections, which makes applying the standard procedure even more difficult. Network reconfiguration must consider critical network characteristics to solve optimally. The steps in finding the optimal configuration include several things, namely, creating a valid network model and developing techniques that can handle network configuration changes quickly and accurately. Other steps are calculating power flow accurately, determining objective functions, determining constraints, and optimizing network configurations.

Several studies have proposed the adoption of distribution network reconfiguration technologies, both conventional and artificial intelligence-based. The proposed methods include fuzzy multi-objective methods, simulated annealing, genetic algorithms, harmony search, ant colony optimization, particle swarm optimization, artificial immune system, social beetle swarm algorithm, and deep learning neural networks. Das [24] and Syahputra et al. [25] proposed a multiobjective fuzzy method approach to solving the optimization problem of radial distribution networks. Jeon et al. [26] applied the simulated annealing method for optimal reconfiguration of large-scale power distribution networks. An approach using genetic algorithms for reconfiguration of distribution networks has been carried out by Enacheanu et al. [27]. Rao et al. [28] proposed the harmony search method to solve the optimization problem of large-scale distribution networks. Furthermore, Falaghi et al. [29] tested the ant colony optimization algorithm to find the most optimal switch combination to improve distribution network performance. The application of the particle swarm optimization (PSO) method to the reconfiguration of the distribution network has been carried out by Niknam et al. [30] and Syahputra et al. $[31,32]$. A system test has been carried out on the IEEE 33-bus model distribution network in the PSO approach. Other artificial intelligence-based methods are the artificial immune system method proposed by Ahmad et al. [33] and the social beetle swarm algorithm method by Chen et al. [34]. An artificial neural network method for optimizing the optimal network configuration has been proposed by $\mathrm{Hu}$ et al. [35] and Zhong et al. [36]. A deep learning method for finding optimal solutions in reconfiguring distribution networks has been proposed by Wang et al. [37], Zheng et al. [38], and Gao et al. [39]. Since network reconfiguration is a multi-objective problem, the adoption of artificial intelligence-based approaches has gained interest. This matter is because methods based on artificial intelligence excel at multi-objective tasks. These goals are accomplished during network reconfiguration by minimizing power losses, optimizing the voltage profile on each bus, minimizing switching operations, and preserving load balance [40]. All these tasks must be completed successfully concurrently.

The purpose of this paper is to discuss how to manage distribution networks, particularly in the presence of dynamically scattered generators. When reconfiguring distribution networks, management of dynamic distribution networks requires a multi-objective strategy. The multi-objective strategy that becomes a significant contribution of this work is to optimize the distribution network configuration with the main target of minimum power losses. Another target that must be achieved simultaneously is the increasing voltage on each distribution network bus, and the load balance for each feeder can be maintained. The method utilized is a sort of clonal selection known as immune-clonal selection (ICS), in which the front of Pareto is not dominating and is equipped with graph theory. The ICS optimization approach is designed to imitate the human immune system's recognition, memory, and adaptive learning capabilities. According to literature reviews [41], the ICS technique is more robust than the GA and PSO methods. It searches the whole search space for solutions, avoiding premature convergence to local minimums. The ICS approach is equipped with graph theory, namely Prim's algorithm, in this suggested research. This strategy is advantageous for generating trees with the 
fewest possible branches throughout the evolutionary process. This approach has the potential to increase the performance of ICS computing significantly.

\section{2- Distribution Network with Scattered Generators (SG) Integration}

Historically, electricity has been generated at power plants using energy sources located in urban or industrial areas. Electrical energy is transported from this plant to load centres, referred to as substations. The energy is subsequently distributed to consumers via the electricity distribution network. Small to medium-scale power production technology has been extensively used in its development. These power facilities are typically composed of renewable energy generators called scattered generators (SG) [25].

The introduction of renewable energy power plants was prompted by several causes, including the advancement of renewable energy generation technology year after year and the intensification of environmental concerns. There are various advantages to implementing renewable energy power plants, including being created by individuals or community organizations, using non-renewable fuel, and being exceptionally environmentally beneficial. This type of power plant is critical in today's industrialized world.

In a classical power system, electrical energy is generated by a power plant in one location and then transmitted through a high, extra-high, or ultra-high voltage transmission network to load centers for distribution to consumers through a distribution network. The large-scale power plants in the electric power system are located in scattered locations in various locations, generally far from load centers. However, these power plants are usually connected to a transmission network and not a distribution network. This task aims to improve the system's effectiveness and efficiency because by connecting directly to the transmission network, losses will be suppressed. In the last two decades, there has been a phenomenon of the growth of small-scale power plants, which are less than $10 \mathrm{MW}$, spread in various places. These power plants generally come from renewable energy sources, often referred to as scattered generators (SG). SG continues to grow and prosper along with the increase in renewable energy technology and the engineering of supporting equipment such as rectifiers, inverters, batteries, and voltage controllers. SGs are generally connected to the electric power distribution network. The advantages of SG are relatively low cost, relatively small risk, and close to the load, which are beneficial in increasing the voltage profile and reducing power losses in the distribution network. Another advantage is that SG can improve the power quality and reliability of the distribution network by increasing the power supply in several locations. These advantages make SG an essential solution for electrical energy needs in rural areas like those in many parts of Indonesia. The presence of SG also plays a role in delaying the need to increase distribution networks by utilizing renewable energy resources from the local potential of each region.

However, the SG may introduce various complications, such as reactive power addition to the network, protection, power conditioning, power quality, and electricity prices. Certain scattered generation technologies, such as wind turbines employ asynchronous (induction generators) that draw reactive electricity from the grid. This device results in a reactive power shortage, either locally or globally, and, as a result, a poor voltage profile, which eventually results in a voltage collapse. Additionally, SG generates more intricate protective measures in the distribution network in terms of selectivity and coordination. The reasons for the distribution network's power flow with SG units are no longer unidirectional, and the error rate has altered. Additionally, several SG technologies, such as photovoltaics and fuel cells, lack a backspin that allows them to respond rapidly to changes in the electrical power balance.

There are four distinct forms of SG, depending on the technology and characteristics of the power source [31]:

- Type I: Direct energy generation injects just active power (P), for example, solar photovoltaics.

- Type II: inject just reactive power (Q), for example, synchronous compensators.

- Type III: SGs, such as asynchronous generators, inject active power but absorb reactive power. $Q E R=(0.5+$ $0.04 P 2)$ is the reactive power absorbed by an asynchronous generator.

- Type IV: SGs, such as synchronous generators, inject both active and reactive power.

Solar photovoltaics and wind power plants are the two types of SG units that operate on the radial distribution network in our study. Solar photovoltaics' SG injects active power, whereas wind power plants' SG inject both active and reactive electricity.

\section{3- Immune-Clonal Selection (ICS)}

\section{3-1-Immune-Clonal Selection Algorithm}

The Immune-Clonal Selection (ICS) principles are used to explain the immune system's fundamental reaction to antigen activation [21], as illustrated in Figure 1. The ICS is a multicellular organism composed of molecules and cells. The system is an organism that functions as an identification mechanism that can supply and resist the malfunction of 
human cells and external pathogenic germs. These bacteria' action recognizes a nearly infinite variety of foreign cells and chemicals, distinguishing them from native, non-infectious cells. B lymphocytes produce antibodies in the body in response to antigens. Each cell secretes a distinct form of antibody that is antigen-specific. Antigens bind to these antibodies, which act as cell receptors. B cells are stimulated to divide and multiply in conjunction with a second signal from accessory cells such as T-helper cells.

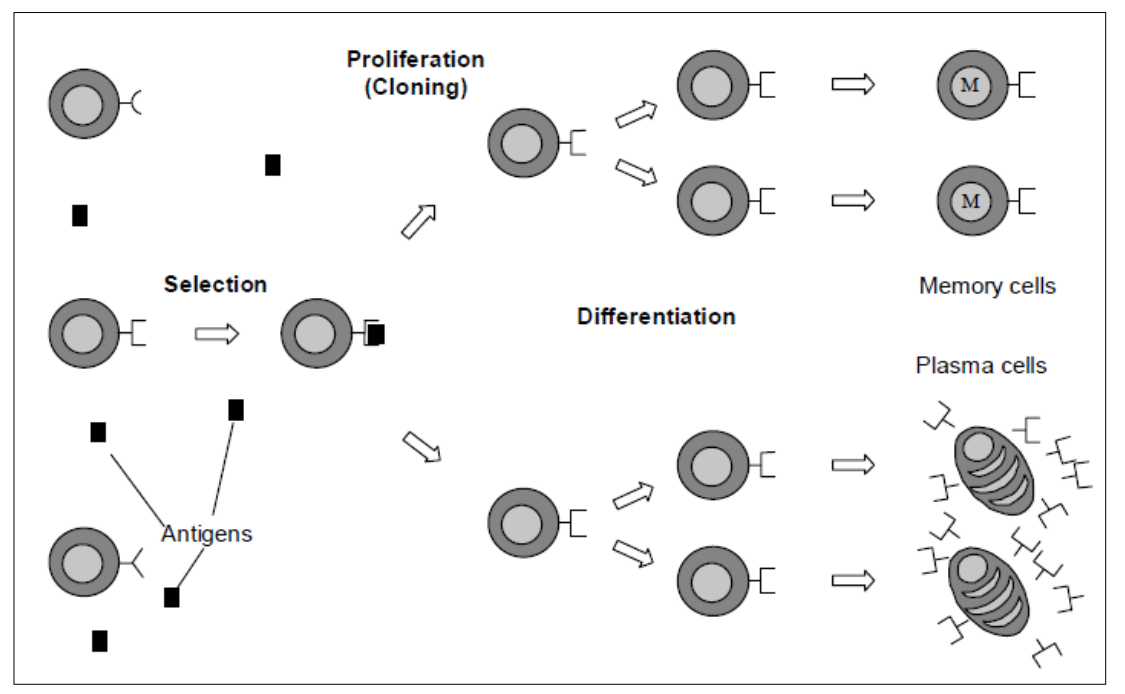

Figure 1. Principle of the immune-clonal selection [42].

Additionally, B cells mature and become plasma cells, which secrete non-individual cell terminal antibodies. Cell proliferation occurs by division, which results in clones, which are cells or groups of cells that are the descendants of a single cell. Additionally, lymphocytes differentiate into plasma cells and then into long-lived memory B cells. This intricate immune system has been adapted to serve as a computing system for multi-objective optimization. This ICS technique offers a few novel properties that make it particularly well-suited for solving multi-objective optimization problems. This ICS technology has been applied to the disciplines of robust control, adaptive control, robotics, optimization, gradient-based systems, and multi-agent systems for neural network-based solutions, image classification, data mining, and optimal delivery in distribution systems. The ICS technique is used because it offers the following benefits: uniqueness, anomaly detection, stranger recognition, memory and learning reinforcement, and pattern recognition. These characteristics enable ICS to identify solutions of good quality and avoid the trap of local minima in computation. Additionally, the ICS approach can resolve difficulties that have never been encountered before.

The affinity between an antibody and an antigen can be established using various ways, including matching rules and a distance measure. The Euclidean distance is a frequently used technique that works well when dealing with real-valued vector representations. To illustrate how ClonalG works using Euclidean distance, let $\mathrm{Ag}=\mathrm{Ag}_{1}, \mathrm{Ag}_{2}, \ldots, \mathrm{Ag}_{\mathrm{N}}$ denote the antigen and antibody sets, respectively, and $A b=A b_{1}, A b_{2}, \ldots, A b_{N}$ denote the support of the order. The degree of correspondence between $\mathrm{Ag}$ and $\mathrm{Ab} \mathrm{d}$ can then be computed using the Euclidean distance:

$$
d=\sum_{i=1}^{N}\left(A g_{i}-A b_{i}\right)^{2}
$$

Additionally, $\mathrm{d}$ is compared to the threshold $\lambda$, and a matching error $\mathrm{E}$ is calculated using the following equation:

$\mathrm{E}=\mathrm{d}-\lambda$

If $\mathrm{E}>0$, it is assumed that the two vectors do not correspond, so the antibody does not identify these antigens. If $\mathrm{E}=$ 0 , the antibody recognizes the antigen. The range of values used to calculate affinity is often between 0 and 1 . The ClonalG algorithm for affinity maturation assumes that the $\mathrm{n}$ greatest affinity antibodies are sorted first and that the number of clones generated for all $\mathrm{n}$ selected antibodies is determined by:

$$
N c=\sum_{i=1}^{n} \operatorname{round}\left(\frac{\alpha \cdot N}{i}\right)
$$

where $N_{c}$ is the clone factor that specifies the scaling factor for the number of antigen clones. $\alpha$ is the most frequently used value, which is $\hat{t} \alpha \in(0,1]$. The total of antibodies is denoted by $\mathrm{N}$. The round () operator spins the argument to its nearest integer value. For instance, if $N=100$ and $\alpha=1$ is used, the antibody with the highest affinity $(i=1)$ will generate 100 clones. In comparison, the antibody with the second-highest affinity will generate 50 clones, and so on [42]. 
Figure 2 illustrates the method of immune-clonal selection for multi-objective optimization used in this study to reconfigure the distribution network.

\section{3-2-Immune-Clonal Selection Algorithm for Multi-objective Optimization of Distribution Network}

The immune-clonal selection (ICS) method is used in this study to reconfigure the distribution network utilizing four different types of objective functions to improve the electrical power distribution system's performance, namely:

- Functions to minimize real power loss.

- Minimize bus voltage variation with these functions.

- Minimization functions for the branch current index.

- Feeder load balancing functions.

ICS is a form of computer intelligence inspired by the vertebrate immune system that has become a prominent way for tackling a variety of optimization issues. The population $(\mathrm{x})$ is a critical variable in the immune-clonal selection method, and population mutation is implemented using the following equation:

$$
X_{i+m j}=X_{y}+N\left(0, \alpha\left(X_{j \max }-X_{j \min }\right)\left(f_{1} / f_{\max }\right)\right.
$$

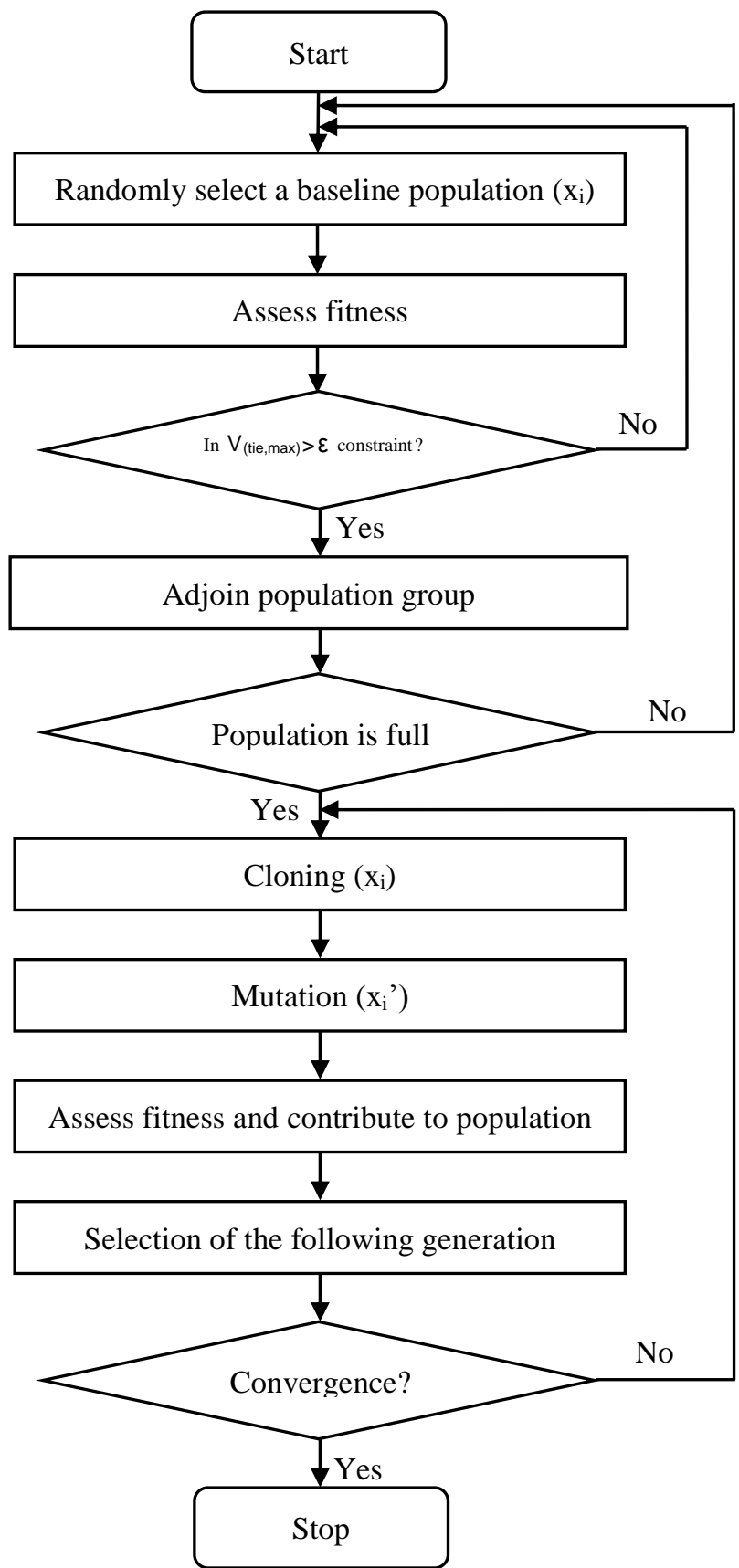

Figure 2. The procedure of ICS method. 
The stages of solving optimization problems with an immune-clonal selection algorithm are as follows:

Step 1: Establish a baseline population

The combination of tie switches on the distribution network should be specified in advance to minimize power losses. The combination tie swaps are determined at random. The quantity of tie switches is defined by the number of tie switches in the distribution network.

\section{Step 2: Determine each population's fitness value}

To reduce power losses in the distribution system, the fitness of the ICS is treated as a total loss. Total losses were determined using the solution of the power flow program. This loss is accomplished by invoking the power flow program as the primary program in the ICS. Additionally, optimizations take into account the voltage limits in the distribution system, ensuring that the minimum and maximum voltages are not exceeded.

\section{Step 3: Cloning process}

The cloning procedure is carried out by combining tie switches and total power losses in the distribution network.

Step 4: The mutation process

Through the use of mutation carriers, the value of cloning is modified in this process. To generate offspring from each parent, mutations on only the operator variations are used. By invoking the power flow algorithm, the fitness of the offspring is determined.

Step 5: The selection procedure

The selection procedure is carried out using priority-based selection algorithms.

Step 6: Convergence is verified

The test procedure is used to decide when the convergence criterion optimization process should come to an end. The difference between the maximum and least fitness values must be smaller than 0.0001 . If the convergence criterion is not satisfied, the operation will be repeated until it is satisfied.

maximum $_{\text {fitness }}-$ minimum $_{\text {finess }} \leq 0.0001$

Figure 2 illustrates the flow chart of the ICS algorithm for optimizing the distribution network configuration.

\section{3-3- Formulation of a Problem}

The primary objectives of network reconfiguration are to minimize active power losses and to enhance the voltage quality. Confined by load flow equations, upper and lower bus voltage constraints, and upper and lower line current limits, the configuration optimization issue is constrained. The following formula can be used to formulate configuration optimization for the purpose of minimizing power loss and maximizing voltage magnitude [31]:

$$
\min P_{\text {loss }}=\sum_{i=1}^{N_{k}} R_{i} \frac{\left(P_{i}^{2}+Q_{i}^{2}\right)}{V_{i}^{2}}
$$

Subject to:

$$
\begin{aligned}
& \mathrm{F}(\mathrm{x})=0 \\
& V_{i, \text { min }} \leq V_{i} \leq V_{i, \text { max }} \\
& I_{i, \text { min }} \leq I_{i} \leq I_{i, \text { max }}
\end{aligned}
$$

where $P_{\text {loss }}$ denotes the cost of active power loss; $N_{k}$ is the branch count; $R_{i}$ is the resistance at the i-th bus; $P_{i}$ and $Q_{i}$ are the active and reactive power that flows out of the bus, respectively. $V_{i}$ denotes the magnitude of the voltage on the i-th bus; $V_{i, \min }$ and $V_{i, \max }$ denote the lower and upper voltage limits on the i-th bus, respectively; $I_{i}$ is the magnitude of the current at bus i-th; and $I_{i, \min }$ and $I_{i, \max }$ are the bus lower and higher current limits, respectively.

\section{4- Results and Discussion}

This research explores two test electrical distribution systems: an IEEE model distribution network 33-bus and an IEEE model distribution network 71-bus. In the assumption, the two test systems have been incorporated into SG. Matlab software was used to implement the ICS approach for reconfiguring a distribution network with SG integration. Two types of distributed generation (SG) are modeled in our work, namely solar photovoltaics and wind farms. SG is 
considered to operate in a steady-state. Thus, solar photovoltaics' SG injects just active power, whereas wind farms' SG injects both active and reactive electricity.

\section{4-1-IEEE Distribution Network 33-Bus Test System}

The first test case in this study is applying the immune-clonal selection (ICS) algorithm to the IEEE model distribution network 33-bus. This distribution network model is widely used by researchers who are pursuing research in the distribution network field. The network model can be regarded as a standard model that is used as a reference for researchers to test the algorithm developed to improve the performance of the electric power distribution network. The voltage system in this radial topology network is $12.66 \mathrm{kV}$, with the number of distribution transformers represented by 33 buses and 32 sections. The IEEE model distribution network 33-bus is shown in Figure 3. As shown in Figure 3, the central grid is a power transformer with a capacity of $10 \mathrm{MVA}$, which acts as the main feeder. Then three lateral feeders supply electrical loads. The power transformer capacity of $10 \mathrm{MVA}$ and the system voltage of $12.66 \mathrm{kV}$ are used as the basis for power analysis. Concerning network reconfiguration, the disconnecting switch plays a significant role. This disconnecting switch is divided into two types, namely tie switches and sectional switches.

The 33-bus distribution network in this study has five tie switches and 32 sectional switches. Tie switches are switches that, in operational conditions, are always open or called ordinarily open. In contrast, sectional switches are switches that, in operational conditions, are always closed or called customarily closed. This distribution network serves a total electrical load of $3715 \mathrm{~kW}$ spread over 33-buses, with details of load and network data [32]. There are four scenarios applied in this study:-the original 33-bus network condition, the 33-bus network condition was reconfigured, the original 33-bus network condition installed three SG units, and the reconfigured 33-bus network condition installed three SG units. Optimal reconfiguration in this study uses the immune-clonal selection (ICS) method with numerous antibodies of 50 and a maximum iteration of 1000 . Optimization is emphasized on reducing active power losses to a minimum with the voltage magnitude maintained in the range of 0.9 to 1.0 p.u. and balanced load conditions on each feeder. An IEEE model distribution network 33-bus was initially configured with five tie switches, namely switches 33, 34, 35, 36, and 37, as illustrated in Figure 3.

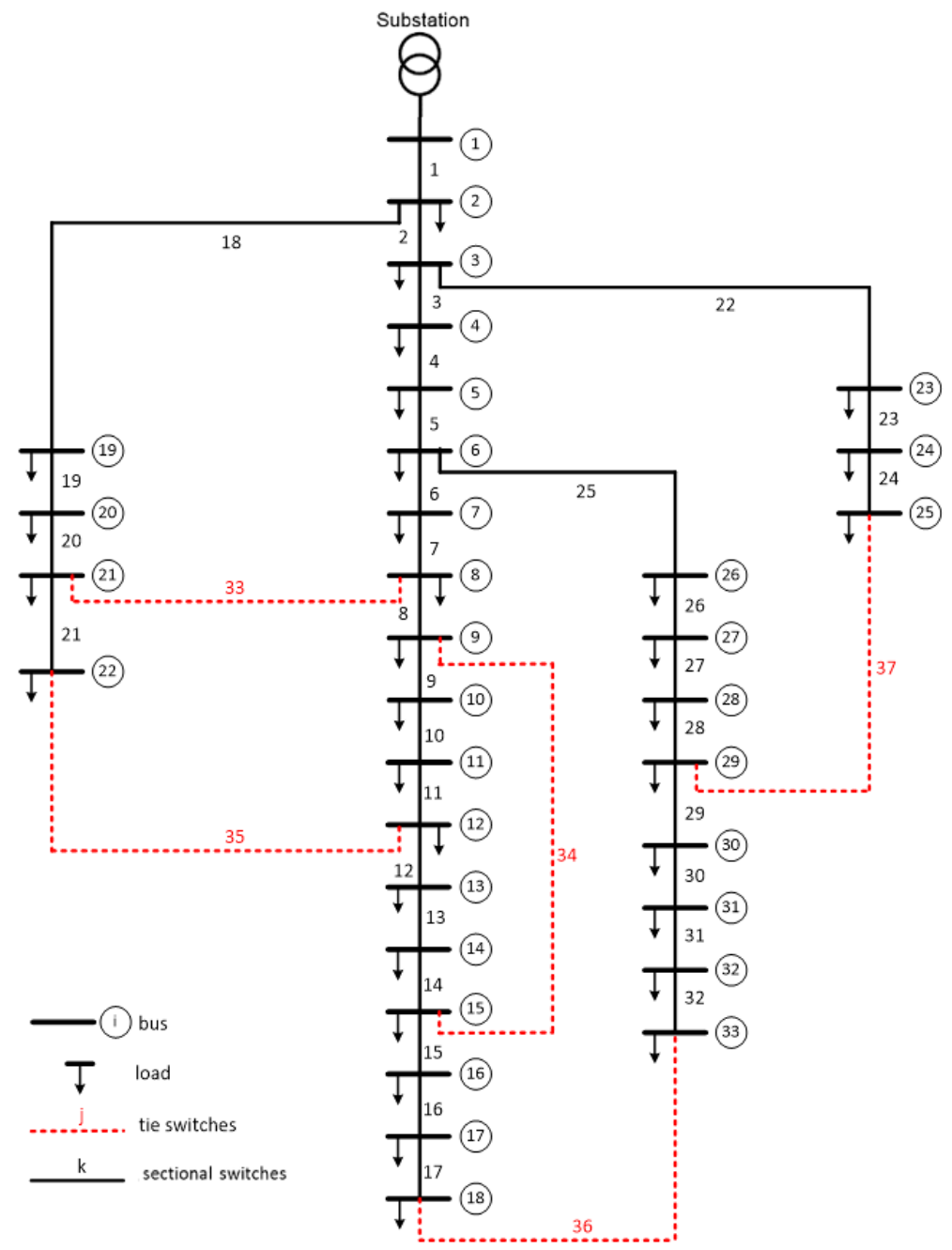

Figure 3. The initial configuration of the IEEE model distribution network 33-bus. 
Table 1. Location and capacity of SG in IEEE model distribution network 33-bus.

\begin{tabular}{clccc}
\hline Name & \multicolumn{1}{c}{ SG type } & Capacity (MW) & Power Factor & Bus Number \\
\hline SG1 & Wind power plant & 2.10 & 0.85 & 18 \\
SG2 & Solar photovoltaic & 1.88 & 1 & 22 \\
SG3 & Wind power plant & 2.22 & 0.85 & 33 \\
\hline & Total & 6.20 & - & - \\
\hline
\end{tabular}

According to the scenario presented in this study, the following stage is to install the SG on the IEEE model distribution network 33-bus. According to the scenario presented in this study, the following stage is to install the SG on the IEEE model distribution network 33-bus.

Three SGs are injected into the 33-bus distribution network: a wind power plant with a capacity of $2.10 \mathrm{MW}$ on bus 18 , a solar photovoltaic with a capacity of $1.88 \mathrm{MW}$ on bus 22, and a wind power plant with a capacity of $2.22 \mathrm{MW}$ on bus 33, as stated in Table 1. Wind power plants with capacities of $2.10 \mathrm{MW}$ and $2.22 \mathrm{MW}$ each have a power factor of 0.85 since they are typically equipped with asynchronous generators. With a capacity of $1.88 \mathrm{MW}$, solar photovoltaic systems have a power factor of unity. Figure 4 illustrates the installation of three SGs in an IEEE model distribution network 33-bus. Based on the network design illustrated in Figure 4, the immune-clonal selection (ICS) approach is used for multi-objective optimization to determine the ideal configuration to achieve low power losses and a decent voltage profile with a balanced load. Figures 5 to 8, and Table 2 clearly illustrate the optimization outcomes.

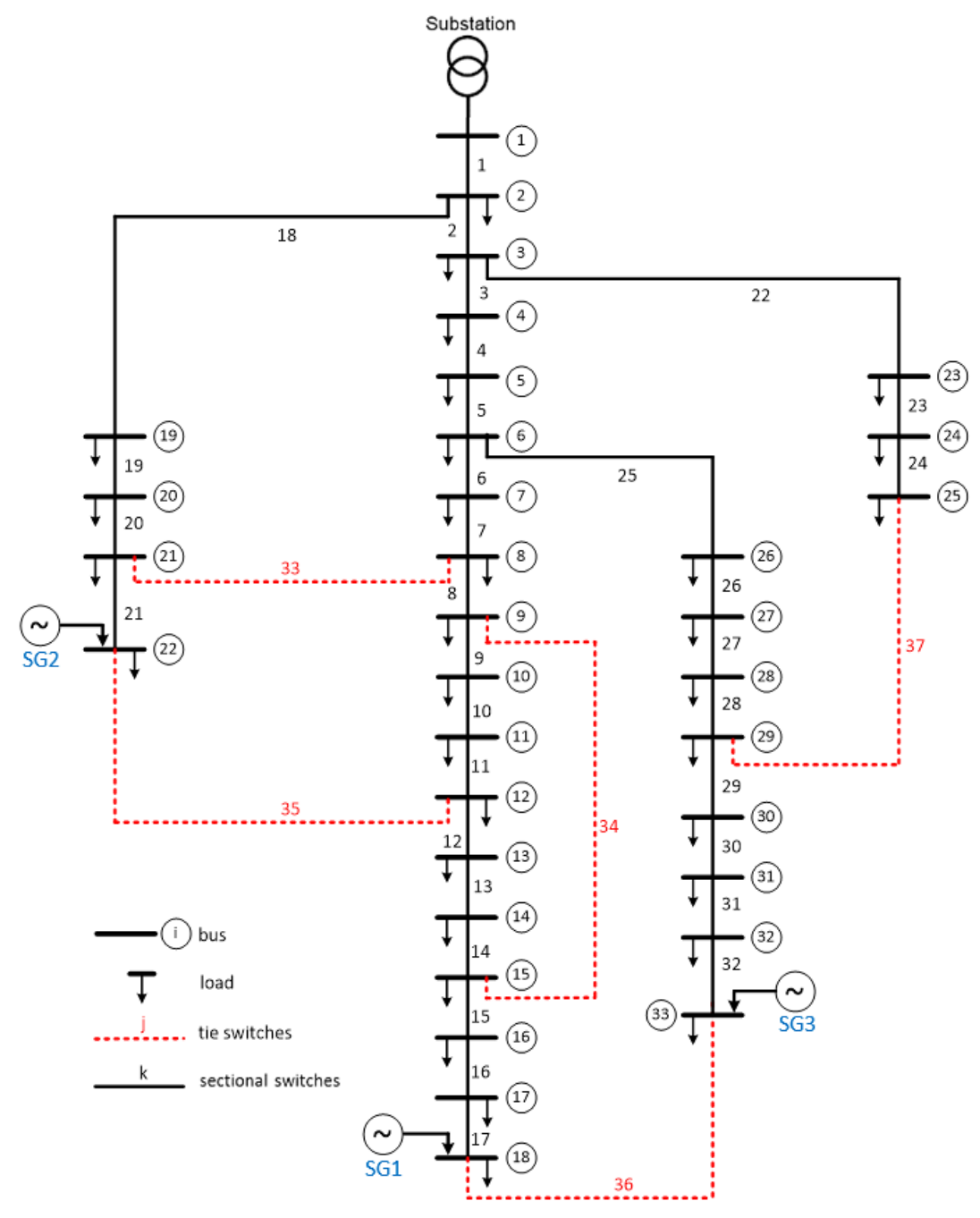

Figure 4. Installation of three SGs on IEEE model distribution network 33-bus.

Figure 5 illustrates the optimization technique for the IEEE model distribution network 33-bus using the ICS approach. The change in power loss during an iteration of the 33-bus network model optimization procedure exemplifies this optimization process. The scenario depicted in Figure 5 (a) is for optimizing the original network, whereas the 
scenario depicted in Figure 5 (b) is for optimizing the network with three SGs added. Each illustration displays 11 optimization experiments, one for each iteration of 5, 10, 30, 50, 100, 200, 300, 400, 500, 700, and 1000. As demonstrated in Figure 5 (a), the upgraded original network scenario requires a minimum of 30 iterations lasting 97.12 seconds to get the optimal outcomes, namely $130.73 \mathrm{~kW}$ in power losses. This study conducted tests up to 1000 iteration to ensure that the numbers obtained were indeed global minima.

Additionally, as illustrated in Figure 5 (b), the scenario for the three optimal SG installed networks requires a minimum of 500 cycles lasting 417.85 seconds to achieve the optimal results, which are $28.12 \mathrm{~kW}$ in power losses. As illustrated in Figure 6, switches 8, 19, 27, 34, and 36 serve as tie switches in this optimization scenario. As a result of this optimization, the ideal network topology results in a $28.12 \mathrm{~kW}$ power loss; or $0.76 \%$ of the overall power loss. Compared to the original state of the IEEE model distribution network 33-bus, which loses $202.71 \mathrm{~kW}$ of electricity, the optimized network with these three SGs loses just $86.18 \%$ of its power. The reduction of power losses using the ICS approach has a significant impact on the operation of the electric power distribution network, ultimately resulting in the saving of potentially lost electrical energy.
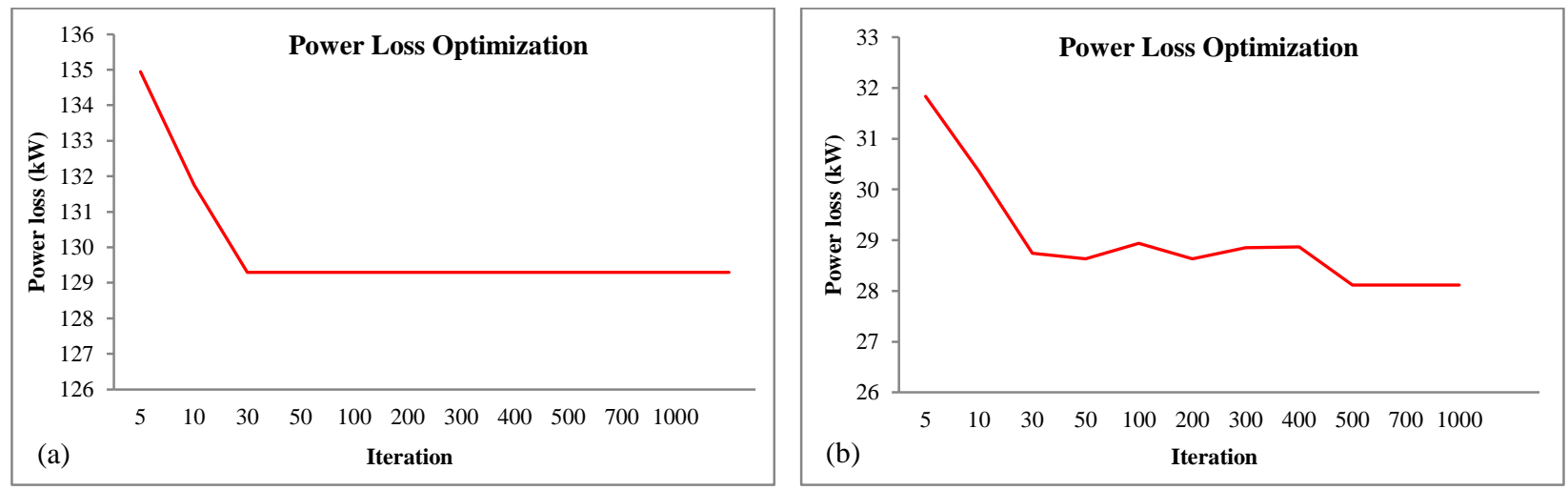

Figure 5. Power loss change to iteration during optimization of IEEE model distribution network 33-bus with three SGs; (a) original network optimized, and (b) SG installed network optimized.

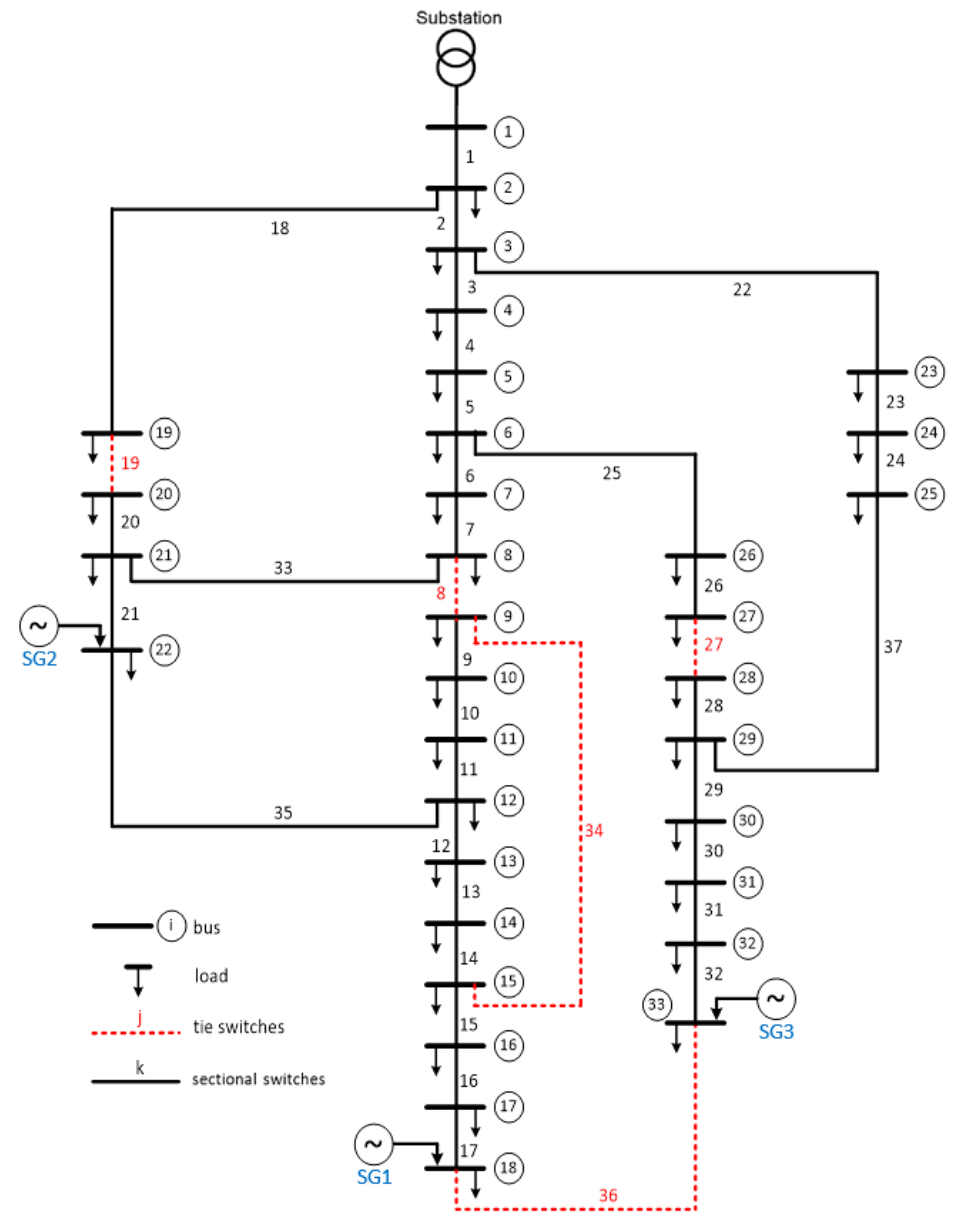

Figure 6. The final configuration of the IEEE model distribution network 33-bus with three SGs. 
Figure 7 illustrates the distribution of power losses across the distribution line components of each bus in the IEEE model distribution network 33-bus test system. Figure 7 depicts four graphs, one for the original network, one for the optimized network, one for the SG installed network, and one for the SG installed network optimized. The green curve demonstrates that the original network has the most significant power loss, while the optimized SG installed network has the lowest power loss, as indicated by the black curve. The distribution of power losses on each bus changes significantly due to the length of the line connecting the buses, the load on each bus, and the voltage profile on each bus. For instance, in each of the four network test scenarios, branch 35 consistently suffers tremendous losses. The branch's power loss was $46 \mathrm{~kW}$ in its original network configuration. This result is because the connection of bus 12 and bus 22 is longer than the line connecting the other branches, and the load served by bus 22 is greater than the load served by the other buses. However, when the ICS approach was optimized, the power losses on branch 35 were reduced to $31 \mathrm{~kW}$. Losses on this branch were reduced to $9 \mathrm{~kW}$ in networks with SG and finally to $8 \mathrm{~kW}$ in optimized networks with SG. As indicated in Table 2, the distribution network power loss was decreased by $35.51 \%$ in the original network optimized scenario, from $202.71 \mathrm{~kW}$ in the original network condition to $130.73 \mathrm{~kW}$ in the optimized condition. The optimized network configuration was obtained by adjusting the tie position. Tie switches 33, 35, and 36 are closed, but sectional switches 6,10, and 32 are open.

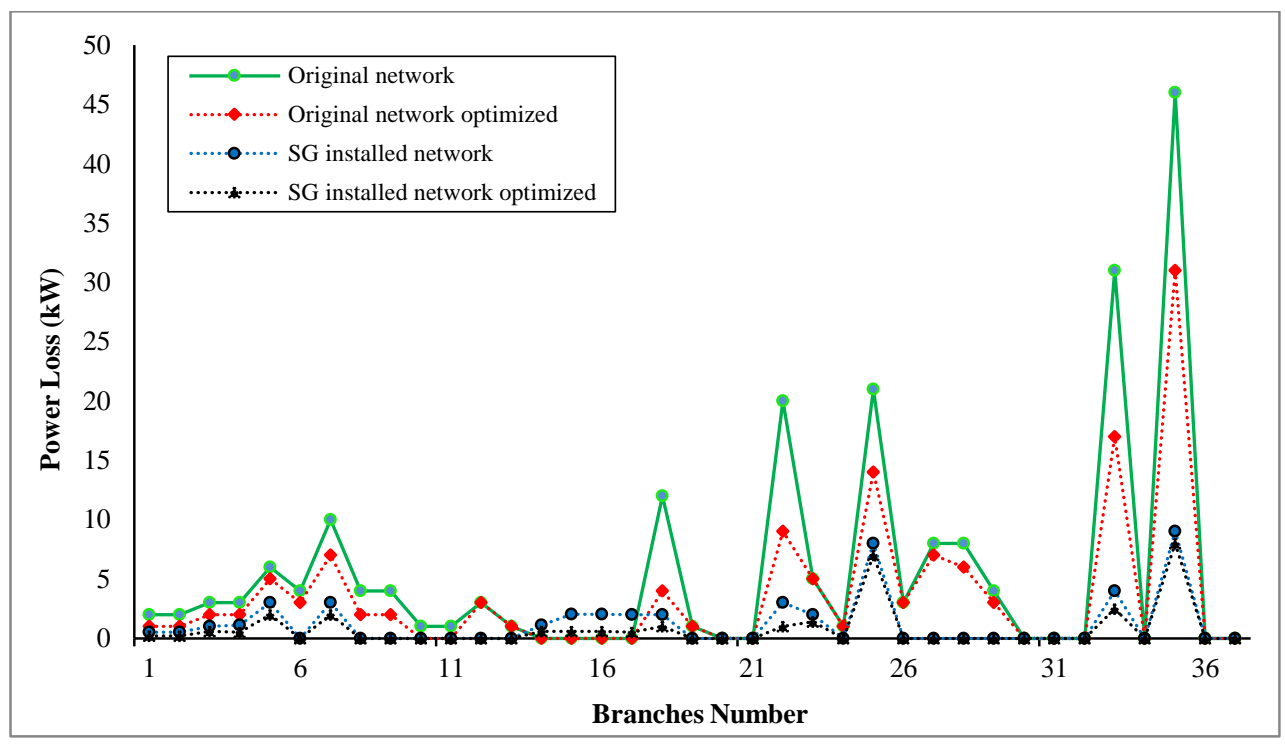

Figure 7. Power loss dispersion of IEEE model distribution network 33-bus.

The installation of scattered generators (SG) on the IEEE model distribution network 33-bus has an essential impact on improving network performance. The voltage profile is getting better, and power losses are decreasing on the buses around the location where the SG is installed. As it can be observed in Figure 7 and Table 1, SG1 is a wind power plant of 2.10 MW with a power factor of 0.85 installed on bus $18, \mathrm{SG} 2$ is a solar photovoltaic type of $1.88 \mathrm{MW}$ with a power factor of unity installed on bus 22, and SG3 is a type of wind power plant of $2.22 \mathrm{MW}$ with a power factor of 0.85 installed on bus 33. For example, the longest line is at branch 35, where the original network condition had a power loss of $46 \mathrm{~kW}$. After installing SG in three locations, the power losses decreased to $9 \mathrm{~kW}$. This significant reduction in power losses was due to the installation of SG2 with a capacity of $1.88 \mathrm{MW}$ on bus 22, which is directly connected to branch 35. The application of the ICS method in optimizing the network configuration resulted in lower power losses at branch 35 , namely $8 \mathrm{~kW}$. Another branch that can be observed a significant change in losses is branch 33 . This branch is directly connected to bus 21, next door to branch 22, where SG2 is installed. In the original network condition, the power loss at branch 33 was $31 \mathrm{~kW}$. The installation of SG2 in bus 22 directly impacts reducing losses in branch 33, which has decreased to $4 \mathrm{~kW}$. The application of the ICS method in optimizing the network configuration causes the loss of power in branch 33 to decrease to $2.5 \mathrm{~kW}$. This review of branches 33 and 35 shows a general picture of the 33-bus distribution network that the installation of SGs has an important influence on reducing distribution network power losses.

Figure 8 shows the voltage profile of the IEEE model distribution network 33-bus in this study. The graph shows four curves in Figure 8, namely the voltage profiles for the original network condition, original network optimized, SG installed network, and SG installed network optimized. The magnitude of the voltage on bus 1 has a maximum value of 1 p.u. or the base voltage of $12.66 \mathrm{kV}$. This voltage magnitude occurs because bus 1 is located close to the power transformer, so there is almost no voltage drop. Furthermore, it can be observed that in the original network voltage profile, the lowest voltage occurs on bus 18 , which is 0.913 p.u. The lowest voltage magnitude on bus 18 occurs because bus 18 is the farthest bus from the power transformer at the substation, so it experiences a significant voltage drop, as shown in Figure 3. Configuration optimization using the ICS method on the original network has succeeded in improving the overall voltage profile. The optimization results show that the lowest voltage shift to bus 32 is 0.947 p.u., as shown 
in Figure 8 and Table 2. The lowest voltage transfer to bus 32 is due to the results of network reconfiguration, which makes bus 32 the farthest bus from the power transformer, as shown in the circuit in Figure 6. However, this lowest voltage is better than the lowest voltage of the original network condition. The highest voltage from the optimization of the network configuration is one p.u. What happens on bus 1 is the same as the original network condition. There is an exciting phenomenon of network configuration optimization results. On bus 20, bus 21 , and bus 22 , the voltage magnitude is lower than the voltage magnitude at the original network condition. This lower voltage is caused by sectional switch 19 being in the open position so that the positions of buses 20, 21, and 22 are farther away from the power transformer than in the original network configuration. However, the voltage drop that occurs is not significant.

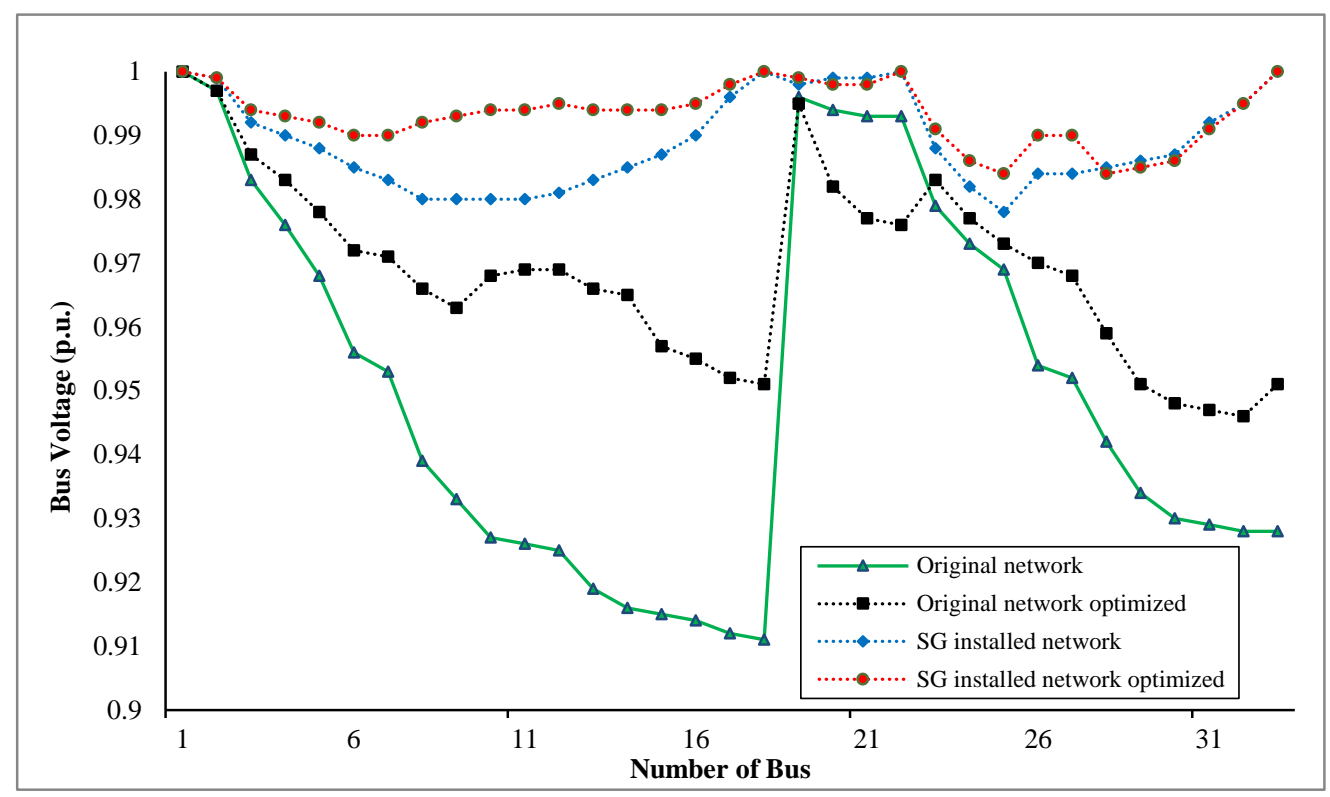

Figure 8. Voltage profile of IEEE model distribution network 33-bus.

The installation of three SGs contributes to improving the voltage profile on the IEEE model distribution network 33bus. This contribution can be further observed in Figure 8. The voltage curve in the SG installed network scenario shows a better profile than the original network condition. The SG installation has succeeded in increasing the network voltage, where the voltage magnitude is one p.u. or $12.66 \mathrm{kV}$ occurred on bus 1 , bus 18 , bus 22 , and bus 33 . The increase in voltage on bus 18 was due to the electric power injection with SG1 installation with a capacity of 2.10 MW with a wind power plant on the bus. Likewise, the increase in voltage on bus 22 is due to the electric power injection with the installation of SG2 with a capacity of $1.88 \mathrm{MW}$ of solar photovoltaic type on the bus. This increase can be observed compared to the original network conditions, namely in the original network conditions, the voltage of bus 18 is 0.913 p.u. and bus 22 is 0.989 p.u. The installation of SG3 with a capacity of $2.22 \mathrm{~kW}$, with a wind power plant type, increased the voltage on bus 33 and nearby buses. The magnitude of the voltage on this bus is 1, wherein the original network condition was only 0.926 p.u. This increase in voltage level was also followed by other buses, which generally experienced a significant increase. In general, the lowest voltage in the SG installed network scenario in this study is of magnitude 0.979 p.u. What happens to bus 25, as shown in Figure 8 and Table 2.

Furthermore, optimizing the IEEE 33-bus distribution network configuration using the ICS method provides a more significant increase in network performance. It can be observed in the voltage profile curve in Figure 8, and almost all buses reach their best voltage profile compared to other scenarios. The lowest voltage magnitude occurs on buses 25 and 28 , which is 0.983 p.u. However, this lowest magnitude is the best voltage profile in this study.

Table 2. Results of IEEE model distribution network 33-bus.

\begin{tabular}{|c|c|c|c|c|c|c|c|c|}
\hline \multirow[b]{2}{*}{ Scenario } & \multicolumn{8}{|c|}{ Variables } \\
\hline & $\begin{array}{l}\text { Power Loss } \\
(\mathbf{k W})\end{array}$ & $\begin{array}{c}\text { Power } \\
\text { Loss }(\%)\end{array}$ & $\begin{array}{c}\text { Power Loss } \\
\text { Reduction }(\%)\end{array}$ & $\begin{array}{c}\text { Lowest Voltage } \\
\text { (p.u.) }\end{array}$ & Highest Voltage (p.u.) & $\begin{array}{l}\text { Tie Switches to } \\
\text { be Closed }\end{array}$ & $\begin{array}{c}\text { Sectional Switches } \\
\text { to be Open }\end{array}$ & $\begin{array}{c}\text { Balanced } \\
\text { Feeder }\end{array}$ \\
\hline Original network & 202.71 & 5.46 & NA & $\begin{array}{c}0.913 \\
\text { (bus } 18)\end{array}$ & $\begin{array}{c}1.00 \\
\text { (bus } 1)\end{array}$ & NA & NA & Yes \\
\hline $\begin{array}{l}\text { Original network } \\
\text { optimized }\end{array}$ & 130.73 & 3.52 & 35.51 & $\begin{array}{c}0.947 \\
\text { (bus 32) }\end{array}$ & $\begin{array}{c}1.00 \\
\text { (bus } 1)\end{array}$ & $\begin{array}{l}33 \\
35 \\
36\end{array}$ & $\begin{array}{c}6 \\
10 \\
32\end{array}$ & Yes \\
\hline $\begin{array}{l}\text { SG installed } \\
\text { network }\end{array}$ & 44.27 & 1.19 & 78.16 & $\begin{array}{c}0.979 \\
\text { (bus 25) }\end{array}$ & $\begin{array}{c}1.00 \\
\text { (bus 1, bus 18, bus 22, bus 33) }\end{array}$ & NA & NA & Yes \\
\hline $\begin{array}{c}\text { SG installed } \\
\text { network optimized }\end{array}$ & 28.12 & 0.76 & 86.18 & $\begin{array}{c}0.983 \\
\text { (bus 25, bus 28) }\end{array}$ & $\begin{array}{c}1.00 \\
\text { (bus 1, bus 18, bus 22, bus 33) }\end{array}$ & $\begin{array}{l}33 \\
35 \\
37\end{array}$ & $\begin{array}{c}8 \\
19 \\
27\end{array}$ & Yes \\
\hline
\end{tabular}


Additionally, the appropriate configuration improves the voltage quality of the IEEE model distribution network 33bus in this study. The results indicated that the ideal configuration could enhance the power distribution system's quality, as seen by the increasing amplitude of the lowest voltage on bus 25 , which is 0.979 to 0.983 p.u. The voltage profile is also increased on practically all buses, excluding those with a voltage magnitude of one p.u., as illustrated in Figure 8. Enhancing the voltage profile and minimizing power losses contribute significantly to the distribution network's performance. As shown in Table 2, the application of the ISC method to the optimization of the IEEE 33-bus network configuration resulted in a power reduction of $35.51 \%$. The installation of three SG units on the network resulted in a decrease in power losses of $78.16 \%$ compared to the original network condition. The most optimal reduction in power losses is obtained in the SG installed network optimized scenario, which is $86.18 \%$. In this most optimal condition, the network power losses are only $28.12 \mathrm{~kW}$ or $0.76 \%$.

The next step is to conduct a comparative study with other methods in optimizing the distribution network configuration in the IEEE model distribution network 33-bus test case to see the performance of this proposed ICS method. The author has conducted a previous study, namely optimization of distribution network configuration using the fuzzy multi-objective method [25] and particle swarm optimization (PSO) methods [31, 32]. Comparison of the optimization results of network configuration with fuzzy multi-objective and PSO methods can be seen in Table 3 and Figure 9.

Table 3. Comparison of optimization results of proposed ICS method with fuzzy multi-objective and PSO methods.

\begin{tabular}{cccccc}
\hline Methods & Test Case Model & SG Injected & $\begin{array}{c}\text { Power Loss } \\
(\mathbf{k W})\end{array}$ & $\begin{array}{c}\text { Minimum Bus } \\
\text { Voltage (p.u.) }\end{array}$ & $\begin{array}{c}\text { Average Bus } \\
\text { Voltage (p.u.) }\end{array}$ \\
\hline Fuzzy multi-objective [25] & IEEE 33-bus & 3 & 30.04 & $\begin{array}{c}0.981 \\
\text { (bus 25) }\end{array}$ & 0.990 \\
PSO [31, 32] & IEEE 33-bus & 3 & 27.63 & $\begin{array}{c}0.983 \\
\text { (bus 24) }\end{array}$ & 0.992 \\
ICS & IEEE 33-bus & 3 & 28.12 & $\begin{array}{c}0.984 \\
\text { (bus 25 and 28) }\end{array}$ & 0.993 \\
\hline
\end{tabular}

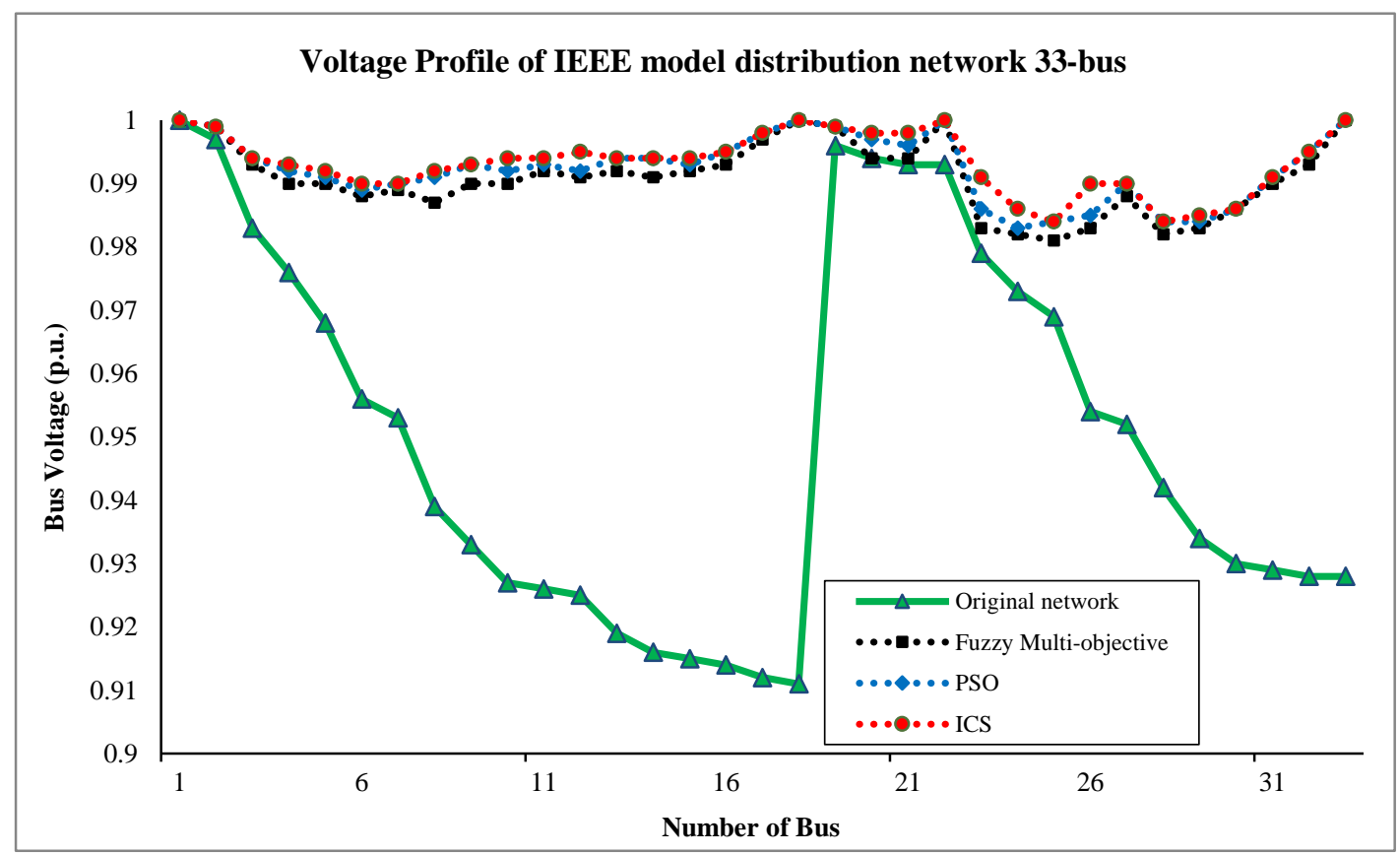

Figure 9. Comparison of voltage profile of the proposed ICS method with fuzzy multi-objective and PSO methods.

As shown in Table 3, the optimization of the IEEE 33-bus network using the fuzzy multi-objective method produces a power loss of $30.04 \mathrm{~kW}$. In comparison, the PSO method produces $27.63 \mathrm{~kW}$, and the proposed ICS method produces $28.12 \mathrm{~kW}$. The PSO method is superior to the ICS and fuzzy multi-objective methods in reducing power losses. However, if we look at the resulting voltage profile, the ICS method's optimization results are superior to the PSO and fuzzy multiobjective methods. The average bus voltage optimized by the ICS method is 0.993 p.u. While the PSO method produces 0.992 p.u. and the fuzzy multi-objective method yields 0.990 p.u. The voltage profile graphically can be seen in Figure 9. It can be seen that the optimization results using the proposed ICS method make the voltage on most buses have a better magnitude than the PSO and fuzzy multi-objective methods. 


\section{4-2-IEEE Distribution Network 71-Bus Test System}

In this section, the second test case is described. The second test case in this study is applying the immune-clonal selection (ICS) algorithm to the IEEE model distribution network 71-bus. This network model is larger than the preceding test case's network model. The voltage system in this radial topology network is $11 \mathrm{kV}$, with the number of distribution transformers represented by 71 buses and 81 sections. The IEEE model distribution network 71-bus is shown in Figure 10. As shown in Figure 10, the primary grid is a power transformer with a capacity of 10 MVA, which acts as the main feeder. Then two lateral feeders supply electrical loads. The power transformer capacity of 10 MVA and the system voltage of $11 \mathrm{kV}$ are used as the basis for power analysis. Concerning network reconfiguration, the disconnecting switch plays a critical role. This disconnecting switch is divided into two types, namely tie switches and sectional switches. The 71-bus distribution network in this study has 11 tie switches and 70 sectional switches. Tie switches are switches that, in operational conditions, are always open or called ordinarily open, while sectional switches are switches that, in operational conditions, are enduringly closed or called customarily closed. This distribution network serves a total electrical load of $4468 \mathrm{~kW}$ spread over 71-buses, with details of load and network data [31]. There are four scenarios applied in this study: the original 71-bus network condition, the 71-bus network condition was reconfigured, the original 71-bus network condition installed four SG units, and the reconfigured 71-bus network condition installed four SG units. Optimal reconfiguration in this study uses the immune-clonal selection (ICS) method with a few antibodies of 50 and a maximum iteration of 1000. Optimization is emphasized on reducing active power losses to a minimum with the voltage magnitude maintained in the range of 0.9 to 1.0 p.u. and balanced load conditions on each feeder. An IEEE model distribution network 71-bus was originally configured with 11 tie switches, namely switches 69, 70, 71, 72, 73, 74, 75, 76, 77, 78, and 79, as illustrated in Figure 10.

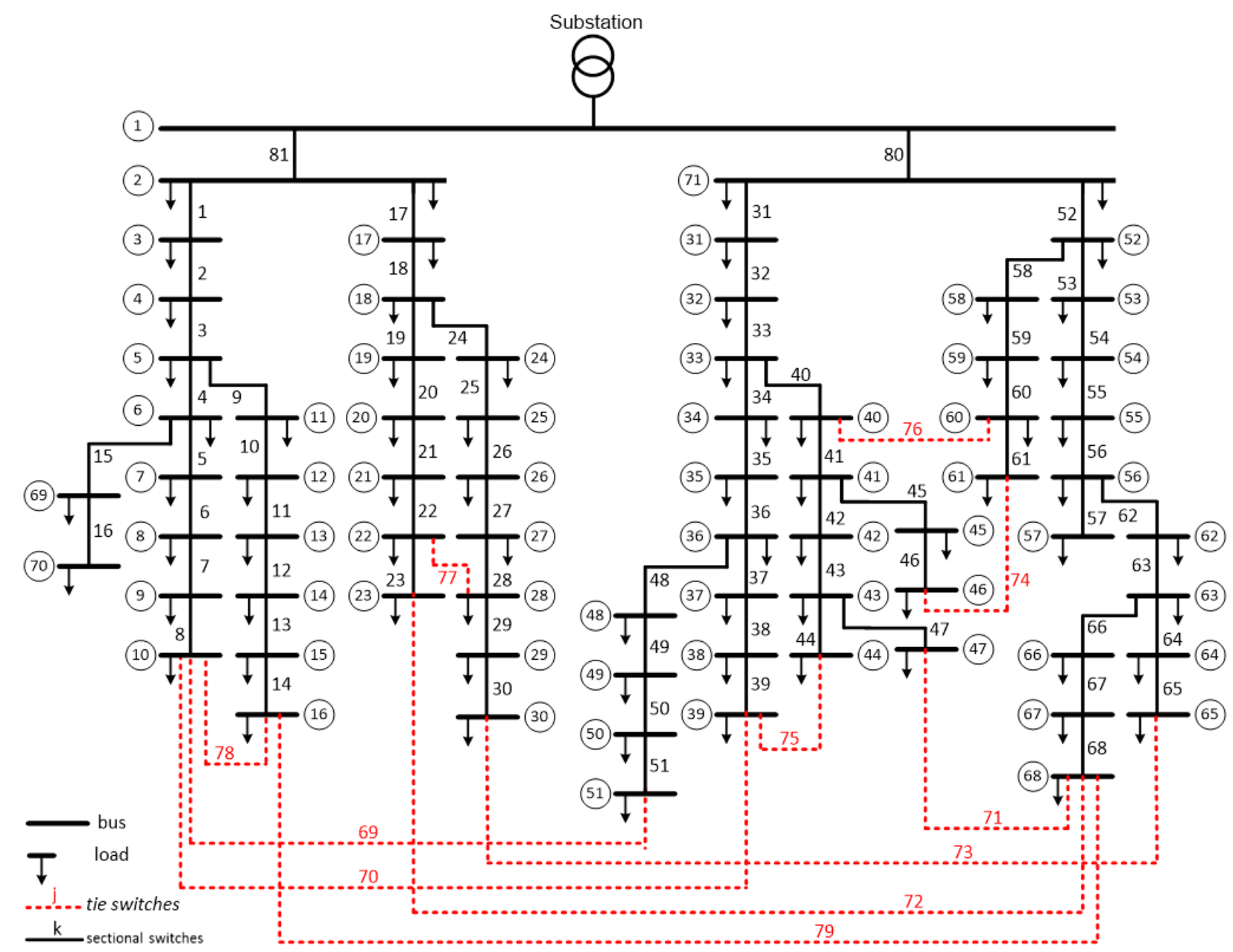

Figure 10. Initial configuration of IEEE model distribution network 71-bus.

According to the scenario presented in this study, the following stage is to install the SG on the IEEE model distribution network 71-bus. Four SGs are injected into the 71-bus distribution network: a wind power plant with a capacity of $0.415 \mathrm{MW}$ on bus 16 , a solar photovoltaic with a capacity of $0.355 \mathrm{MW}$ on bus 29 , a solar photovoltaic with a capacity of $0.250 \mathrm{MW}$ on bus 35, and a wind power plant with a capacity of $0.525 \mathrm{MW}$ on bus 63 , as stated in Table 4. Wind power plants with capacities of $0.415 \mathrm{MW}$ and $0.525 \mathrm{MW}$ each have a power factor of 0.85 since they are typically equipped with asynchronous generators. With capacities of 0.355 and $0.250 \mathrm{MW}$, respectively, the two solar photovoltaic systems have a unity power factor. Figure 11 illustrates the installation of three SGs in an IEEE model 
distribution network 71-bus. Based on the network design illustrated in Figure 11, the immune-clonal selection (ICS) approach is used for multi-objective optimization to determine the ideal configuration to achieve low power losses and a decent voltage profile with a balanced load. Figures 12 to 15, and Table 5 clearly illustrate the optimization outcomes.

Table 4. Location and capacity of IEEE model distribution network 71-bus.

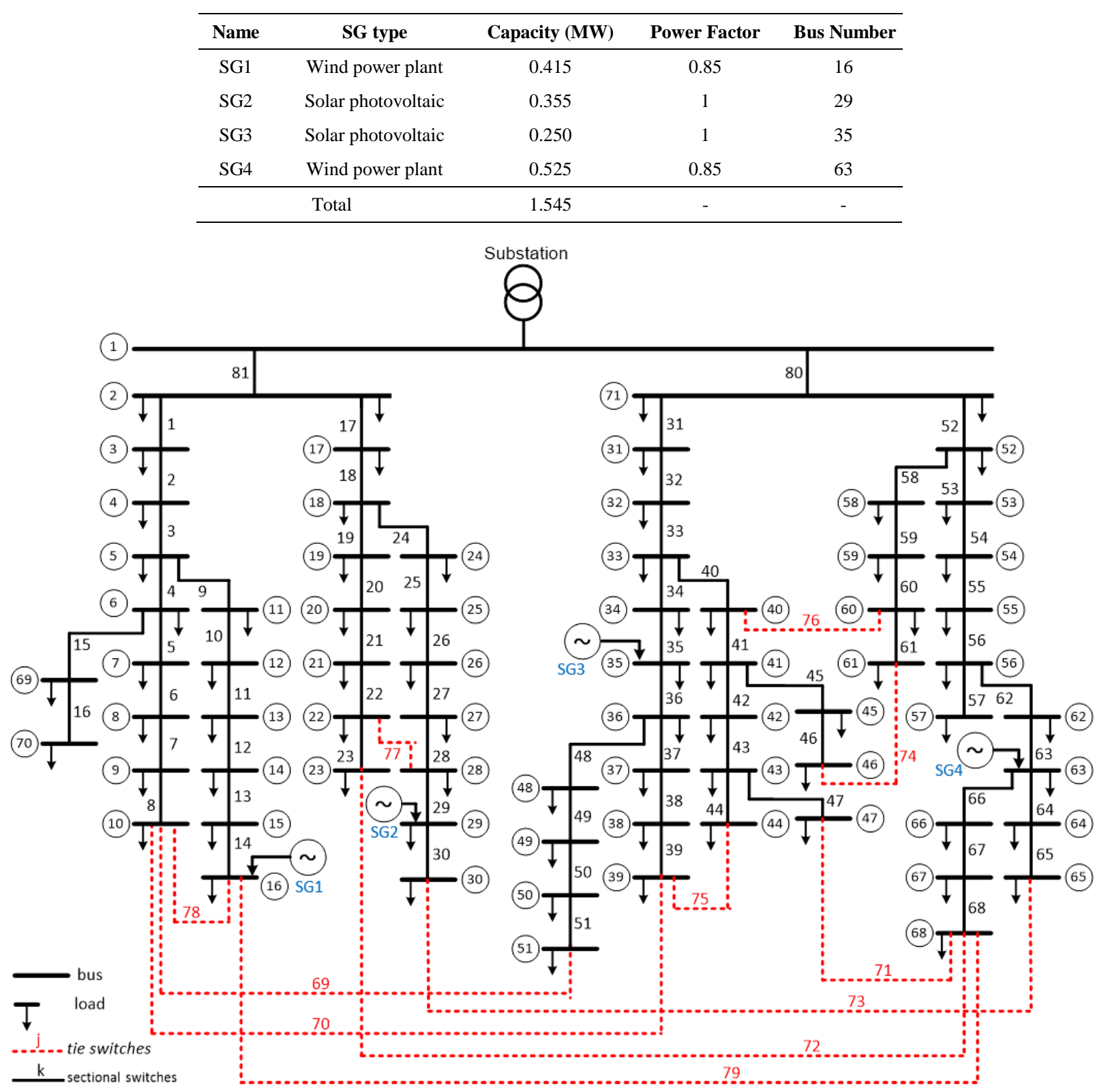

Figure 11. Installation of four SGs on IEEE model distribution network 71-bus.
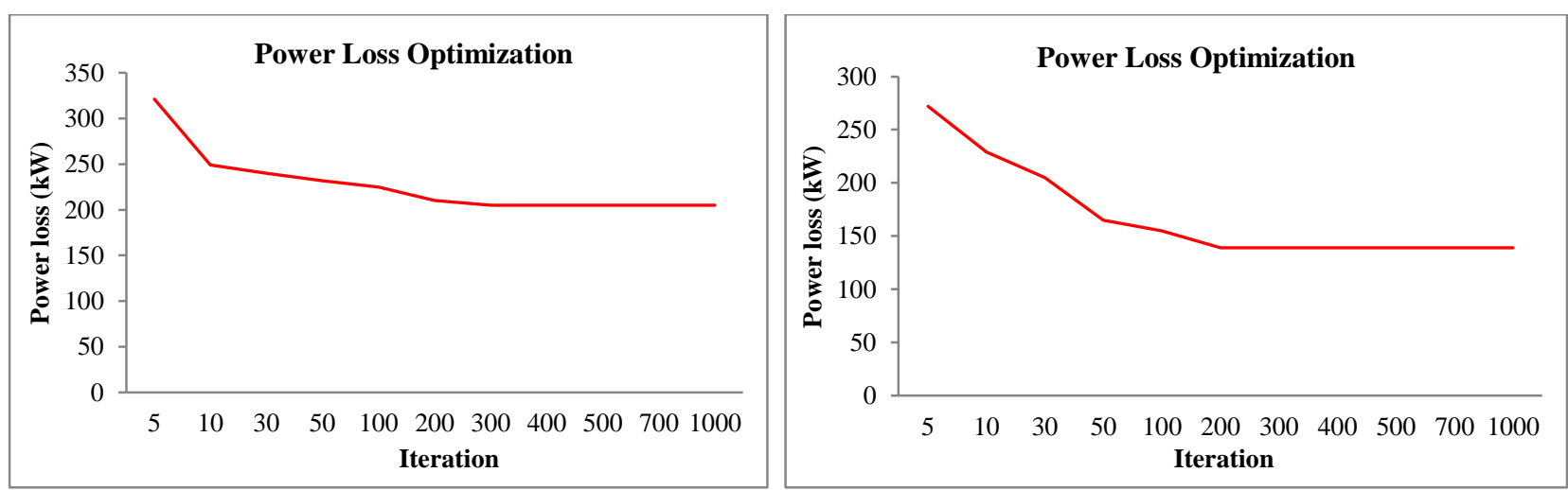

Figure 12. Power loss change to iteration during optimization of IEEE model distribution network 71-bus with four SGs; (a) original network optimized, and (b) SG installed network optimized. 
The IEEE model distribution network 71-bus optimization technique illustrated in Figure 12 is based on the ICS approach. The change in power loss during an iteration of the 71-bus network model optimization procedure exemplifies this optimization process. The scenario depicted in Figure 12 (a) is for optimizing the original network, whereas the scenario depicted in Figure 12 (b) is for optimizing the network with four SGs added. Each illustration displays 11 optimization experiments, one for each iteration of 5, 10, 30, 50, 100, 200, 300, 400, 500, 700, and 1000. As demonstrated in Figure 12 (a), the upgraded original network scenario requires a minimum of 300 iterations lasting 321.56 seconds to get the optimal outcomes, namely $205.17 \mathrm{~kW}$ in power losses. This study conducted tests up to 1000 iteration to ensure that the numbers obtained were indeed global minima.

Additionally, as illustrated in Figure 12 (b), the scenario for the optimal three SG installed networks requires a minimum of 200 cycles lasting 276.24 seconds to achieve the optimal results, which are $138.95 \mathrm{~kW}$ in power losses. As illustrated in Figure 14, switches 5, 20, 42, 49, 60, 68, 70, 71, 73, 76, and 79 serve as tie switches in this optimization scenario. As a result of this optimization, the ideal network topology results in a $138.95 \mathrm{~kW}$ power loss or $3.11 \%$ of the overall power loss. The optimization of the IEEE 71-bus distribution network with four SGs produces minimum active power losses. In this condition, the network power losses are only $138.95 \mathrm{~kW}$ or $3.11 \%$ of the total load power. This reduction in power loss is significant compared to the network's original condition, where the power losses are 228.26 $\mathrm{kW}$ or $5.11 \%$ of the total load power. The results of this optimization show that the reduction of power losses with the ICS approach has a significant impact on the performance of the power distribution network, which in turn saves electrical energy that has the potential to be lost.

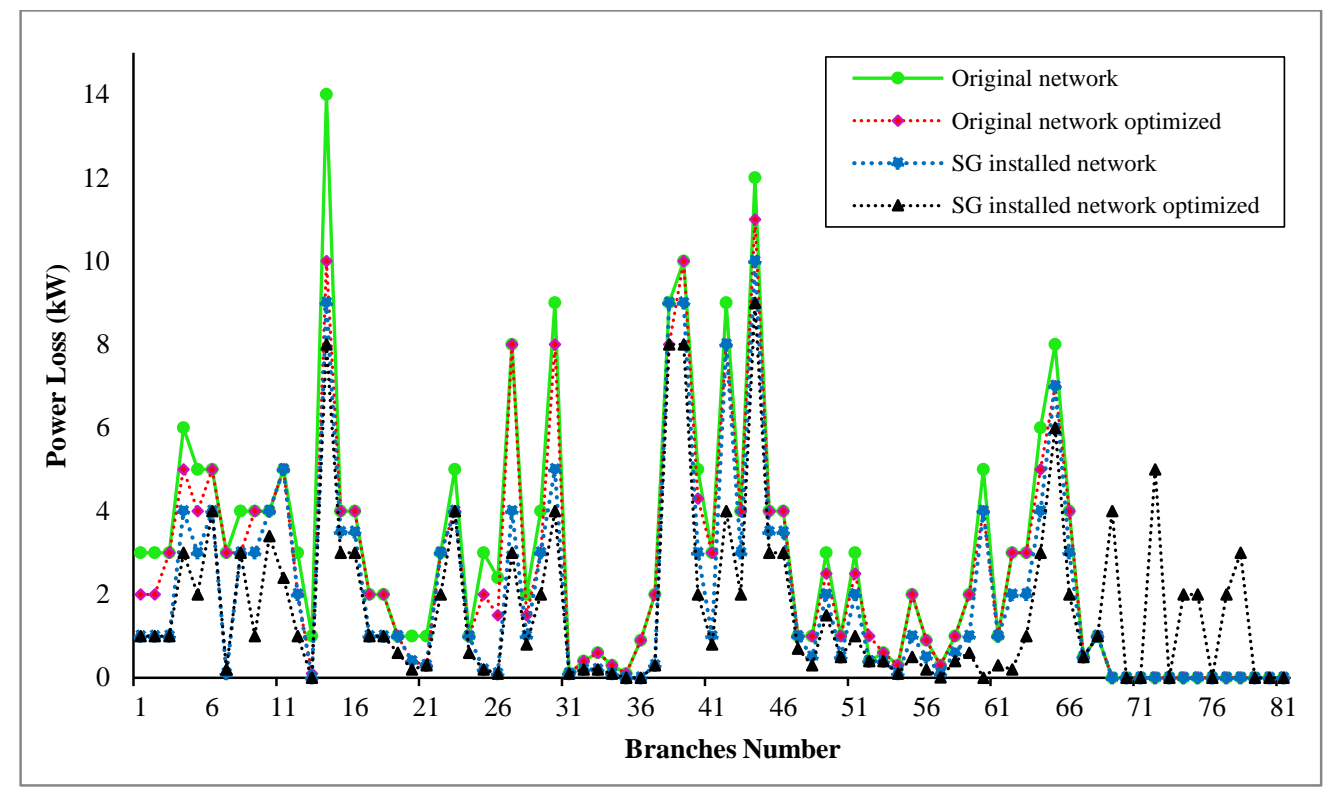

Figure 13. Power loss dispersion of IEEE model distribution network 71-bus.

Figure 13 illustrates the distribution of power losses across the distribution line components of each bus in the IEEE model distribution network 71-bus test system. Figure 13 depicts four graphs, one for the original network, one for the optimized network, one for the SG installed network, and one for the SG installed network optimized. As demonstrated by the green curve, the original network has the largest power loss, while the SG installed network optimized has the lowest power loss, as indicated by the black curve. The distribution of power losses on each bus changes significantly due to the line length connecting the buses, the load on each bus, and the voltage profile on each bus. For instance, in each of the four network test scenarios, branch 14 consistently suffers the greatest losses. The branch's power loss was $14 \mathrm{~kW}$ in its original network configuration. This loss is because the connection of bus 15 and bus 16 is longer than the line connecting the other branches, and the load served by bus 16 is greater than the load served by the other buses. Also, in the original network conditions, bus 16 is one of the most distant buses from the substation. However, when the ICS approach was optimized, the power losses on branch 14 were reduced to $10 \mathrm{~kW}$. Losses on this branch were reduced to $9 \mathrm{~kW}$ in networks with SG and finally to $8 \mathrm{~kW}$ in optimized networks with SG. As indicated in Table 5, the distribution network power loss was decreased by $10.12 \%$ in the original network optimized scenario, from $228.26 \mathrm{~kW}$ in the original network condition to $205.17 \mathrm{~kW}$ in the optimized condition. The optimized network configuration was obtained by adjusting the tie position. Tie switches $69,72,74,75,77$, and 78 are closed, but sectional switches $8,22,42,48,60$, and 68 are open.

The installation of scattered generators (SG) on the IEEE model distribution network 71-bus has an essential impact on improving network performance. The voltage profile is getting better, and power losses are decreasing on the buses around the location where the SG is installed. As it can be observed in Figure 13 and Table 4, SG1 is a wind power plant 
of $0.415 \mathrm{MW}$ with a power factor of 0.85 installed on bus 16 , SG2 is a solar photovoltaic type of 0.355 MW with a power factor of unity installed on bus 29 , SG3 is a solar photovoltaic type of $0.250 \mathrm{MW}$ with a power factor of unity installed on bus 35, and SG4 is a type of wind power plant of $0.525 \mathrm{MW}$ with a power factor of 0.85 installed on bus 63 . The total capacity of the four SGs injected into the 77-bus distribution network is $1.545 \mathrm{MW}$. For example, the longest line is at branch 14, where the original network condition had a power loss of $14 \mathrm{~kW}$. After installing SG in three locations, the power losses decreased to $9 \mathrm{~kW}$. This significant reduction in power losses was due to the SG1 installation with a capacity of $0.415 \mathrm{MW}$ on bus 16 , which is directly connected to branch 14 . The application of the ICS method in optimizing the network configuration resulted in lower power losses at branch 14, namely $8 \mathrm{~kW}$. Another branch that can be observed a significant change in losses is branch 30 . This branch is directly connected to bus 29 , connected to branch 30, where SG2 is installed. In the original network condition, the power loss at branch 30 was $9 \mathrm{~kW}$. The installation of SG2 in bus 29 directly impacts reducing losses in branch 30, which has decreased to $5 \mathrm{~kW}$. The application of the ICS method in optimizing the network configuration causes the loss of power in branch 30 to decrease to $4 \mathrm{~kW}$. This review of branches 14 and 30 shows a general picture of the 71-bus distribution network that the installation of SGs has an important influence on reducing distribution network power losses.

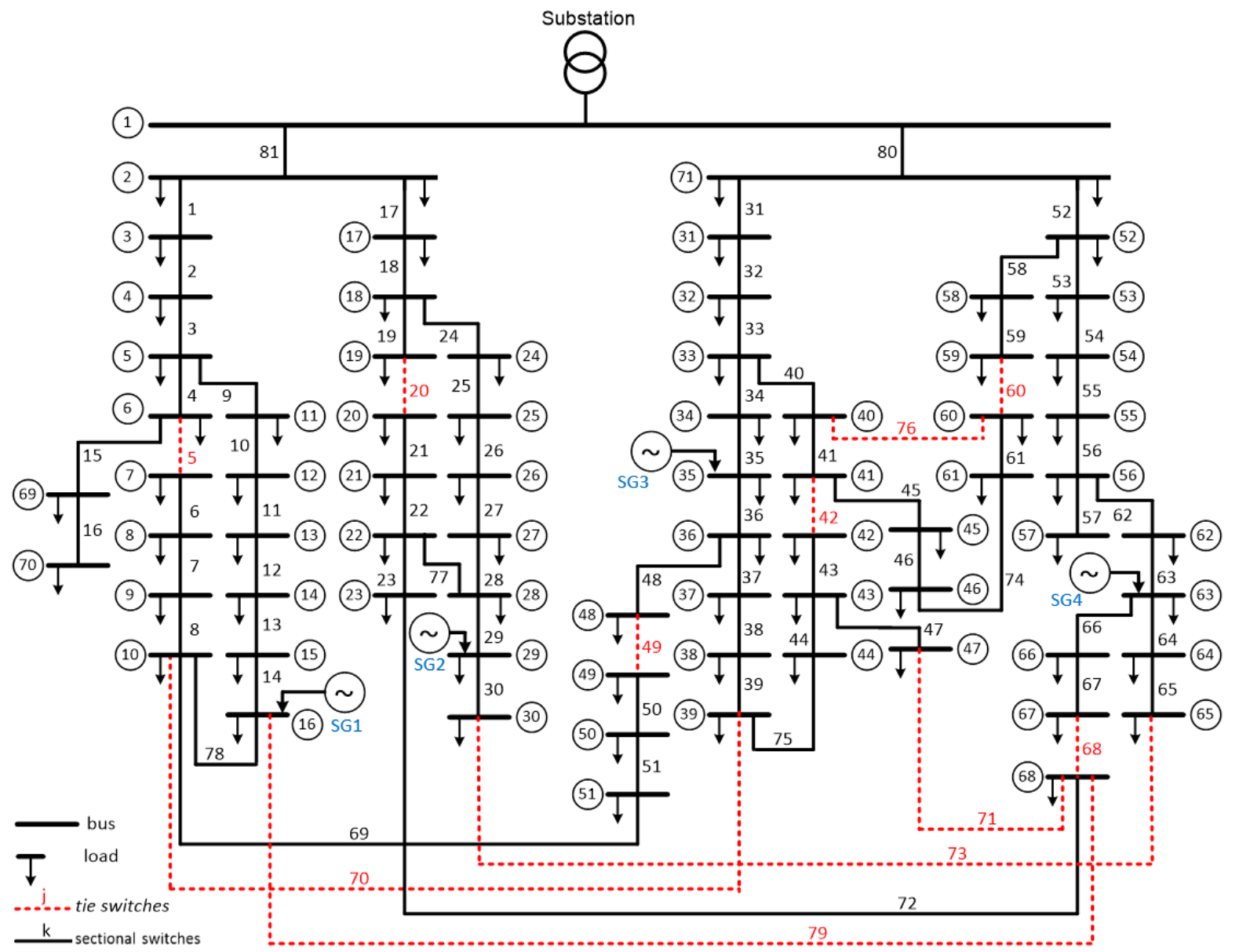

Figure 14. Final configuration of IEEE model distribution network 71-bus with integration of four SGs.

Figure 15 shows the voltage profile of the IEEE model distribution network 71-bus in this study. The graph shows four curves in Figure 15, namely the voltage profiles for the original network condition, original network optimized, SG installed network, and SG installed network optimized. The voltage magnitude on bus 1 has a maximum value of one p.u. or according to the base voltage of $11 \mathrm{kV}$. This voltage is because the buses are very close to the power transformer position at the substation so that there is almost no voltage drop. The voltage magnitude on bus 2 , bus 17 , bus 52 , and bus 71 is also at the best profile, namely one p.u. This voltage is because the buses are very close to the position of the power transformer at the substation, so that there is almost no voltage drop.

Furthermore, it can be observed that in the original network voltage profile, the lowest voltage occurs on bus 68, which is 0.906 p.u. The lowest voltage magnitude on bus 68 occurs because bus 68 is the farthest bus from the power transformer at the substation, so it experiences a critical voltage drop, as shown in Figure 14. Configuration optimization using the ICS method on the original network has succeeded in improving the overall voltage profile. The optimization results show that the lowest voltage on bus 68 is 0.918 p.u., as shown in Figure 15 and Table 5. However, this lowest voltage is better than the lowest voltage of the original network condition. The highest voltage from the optimization of the network configuration is one p.u. 


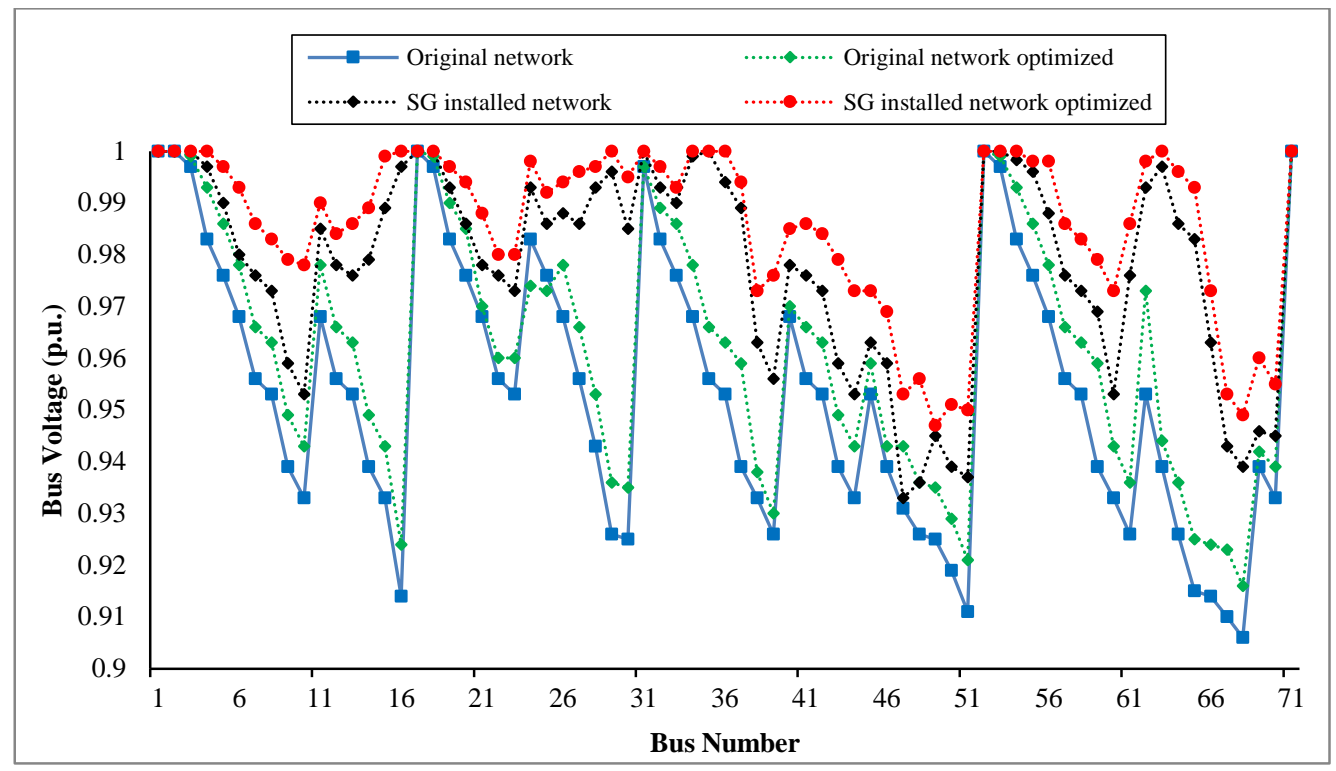

Figure 15. Voltage profile of IEEE model distribution network 71-bus.

Table 5. Results of IEEE model distribution network 71-bus.

\begin{tabular}{|c|c|c|c|c|c|c|c|c|}
\hline \multirow[b]{2}{*}{ Scenario } & \multicolumn{8}{|c|}{ Variables } \\
\hline & $\begin{array}{c}\text { Power Loss } \\
(\mathbf{k W})\end{array}$ & $\begin{array}{c}\text { Power } \\
\text { Loss }(\%)\end{array}$ & $\begin{array}{c}\text { Power Loss } \\
\text { Reduction (\%) }\end{array}$ & $\begin{array}{c}\text { Lowest } \\
\text { Voltage (p.u.) }\end{array}$ & Highest Voltage (p.u.) & $\begin{array}{c}\text { Tie } \\
\text { Switches to } \\
\text { be Closed } \\
\end{array}$ & $\begin{array}{c}\text { Sectional } \\
\text { Switches to } \\
\text { be Open } \\
\end{array}$ & $\begin{array}{c}\text { Balanced } \\
\text { Feeder }\end{array}$ \\
\hline Original network & 228.26 & 5.11 & NA & $\begin{array}{c}0.906 \\
\text { (bus 68) }\end{array}$ & $\begin{array}{c}1.00 \\
\text { (bus } 1 \text {, bus } 2 \text {, bus } 17 \text {, bus } \\
52 \text {, bus } 71 \text { ) }\end{array}$ & NA & NA & Yes \\
\hline $\begin{array}{l}\text { Original network } \\
\text { optimized }\end{array}$ & 205.17 & 4.59 & 10.12 & $\begin{array}{c}0.918 \\
\text { (bus 68) }\end{array}$ & $\begin{array}{c}1.00 \\
\text { (bus } 1 \text {, bus } 2 \text {, bus } 17 \text {, bus } \\
52 \text {, bus } 71 \text { ) }\end{array}$ & $\begin{array}{l}69 \\
72 \\
74 \\
75 \\
77 \\
78\end{array}$ & $\begin{array}{c}8 \\
22 \\
42 \\
48 \\
60 \\
68\end{array}$ & Yes \\
\hline $\begin{array}{l}\text { SG installed } \\
\text { network }\end{array}$ & 161.18 & 3.61 & 29.39 & $\begin{array}{c}0.934 \\
\text { (bus 47) }\end{array}$ & $\begin{array}{c}1.00 \\
\text { (bus } 1 \text {, bus } 2 \text {, bus } 3 \text {, bus } \\
17 \text {, bus } 31 \text {, bus } 34 \text {, bus } 35 \text {, } \\
\text { bus } 52 \text {, bus } 54 \text {, bus } 71 \text { ) }\end{array}$ & NA & NA & Yes \\
\hline $\begin{array}{c}\text { SG installed } \\
\text { network optimized }\end{array}$ & 138.95 & 3.11 & 39.13 & $\begin{array}{c}0.948 \\
\text { (bus 49) }\end{array}$ & $\begin{array}{c}1.00 \\
\text { (bus } 1 \text {, bus } 2 \text {, bus } 3 \text {, bus } \\
16 \text {, bus } 17 \text {, bus } 29 \text {, bus } 31 \text {, } \\
\text { bus } 34 \text {, bus } 35 \text {, bus } 36 \text {, bus } \\
52 \text {, bus } 54 \text {, bus } 55 \text {, bus } 71 \text { ) }\end{array}$ & $\begin{array}{l}69 \\
72 \\
74 \\
75 \\
77 \\
78\end{array}$ & $\begin{array}{c}5 \\
20 \\
42 \\
49 \\
60 \\
68\end{array}$ & Yes \\
\hline
\end{tabular}

The installation of four SGs contributes to improving the voltage profile on the IEEE model distribution network 71bus. This contribution can be further observed in Figure 15. The voltage curve in the SG installed network scenario shows a better profile than the original network condition. The SG installation has succeeded in increasing the network voltage, where the voltage magnitude is 1 p.u. or $11 \mathrm{kV}$ occurred on bus 1 , bus 2 , bus 17 , bus 52 , and bus 71 . If we observe the graph in Figure 15, a significant increase in voltage occurs at bus 16. In the original network condition, the voltage magnitude on bus 16 was 0.913 p.u. The SG1 installation with a capacity of $0.415 \mathrm{MW}$ on bus 16 increases in the voltage magnitude on bus 16 to 0.996 p.u., almost close to 1 p.u. Likewise, the voltage increase on bus 29 is due to the injection of electric power with the installation of SG2 with a capacity of $0.355 \mathrm{MW}$ of solar photovoltaic type on the bus. This increase can be observed compared to the original network conditions, namely in the original network conditions, the voltage of bus 29 is 0.926 p.u., and bus 30 is 0.924 p.u. The installation of SG3 with a capacity of 0.250 $\mathrm{kW}$, with a solar photovoltaic type, increased the voltage on bus 35 and nearby buses. The magnitude of the voltage on this bus is 1 , wherein the original network condition it was only 0.955 p.u. This increase in voltage level was also followed by other buses, which generally experienced a significant increase. In general, the lowest voltage in the SG installed network scenario in this study is of magnitude 0.934 p.u. in bus 47.

Furthermore, optimizing of the IEEE 71-bus distribution network configuration using the ICS method provides a more significant increase in network performance. It can be observed in the voltage profile curve in Figure 15, and almost all buses reach their best voltage profile compared to other scenarios. The lowest voltage magnitude occurs on 
bus 49 , which is 0.948 p.u. However, this lowest magnitude is the best voltage profile in this study.

Additionally, the appropriate configuration improves the voltage quality of the IEEE model distribution network 71bus in this study. The results indicated that the ideal configuration could enhance the power distribution system's quality. As seen by the increasing amplitude of the lowest voltage on bus 47 , which is 0.934 to 0.948 p.u. in bus 49 . The voltage profile is also increased on practically all buses, excluding those with a voltage magnitude of 1 p.u., as illustrated in Figure 15. Enhancing the voltage profile and minimizing power losses contribute significantly to the distribution network's performance. As shown in Table 5, the application of the ISC method to the optimization of the IEEE 33-bus network configuration resulted in a power reduction of $10.12 \%$. The installation of four SG units on the network resulted in a decrease in power losses of $29.39 \%$ compared to the original network condition. The most optimal reduction in power losses is obtained in the SG installed network optimized scenario, which is $39.13 \%$. In this most optimal condition, the network power losses are only $138.95 \mathrm{~kW}$ or $3.11 \%$.

The next step is to conduct a comparative study with other methods in optimizing the distribution network configuration in the IEEE model distribution network 71-bus test case to see the performance of this proposed ICS method. The author has conducted a previous study, namely optimization of distribution network configuration using the fuzzy multi-objective method as can be seen in references [25] and particle swarm optimization (PSO) methods [31, 32]. Comparison of the optimization results of network configuration with fuzzy multi-objective and PSO methods can be seen in Table 6 and Figure 16.

Table 6. Comparison of optimization results of proposed ICS method with fuzzy multi-objective and PSO methods.

\begin{tabular}{cccccc}
\hline Methods & Test Case Model & SG Injected & $\begin{array}{c}\text { Power Loss } \\
(\mathbf{k W})\end{array}$ & $\begin{array}{c}\text { Minimum Bus } \\
\text { Voltage (p.u.) }\end{array}$ & $\begin{array}{c}\text { Average Bus } \\
\text { Voltage (p.u.) }\end{array}$ \\
\hline $\begin{array}{c}\text { Fuzzy multi- } \\
\text { objective [25] }\end{array}$ & IEEE 71-bus & 4 & 142.56 & $\begin{array}{c}0.944 \\
\text { (bus 49) }\end{array}$ & 0.983 \\
PSO [31, 32] & IEEE 71-bus & 4 & 137.23 & $\begin{array}{c}0.946 \\
\text { (bus 49) }\end{array}$ & 0.984 \\
ICS & IEEE 71-bus & 4 & 138.95 & $\begin{array}{c}0.947 \\
\text { (bus 49) }\end{array}$ & 0.986 \\
\hline
\end{tabular}

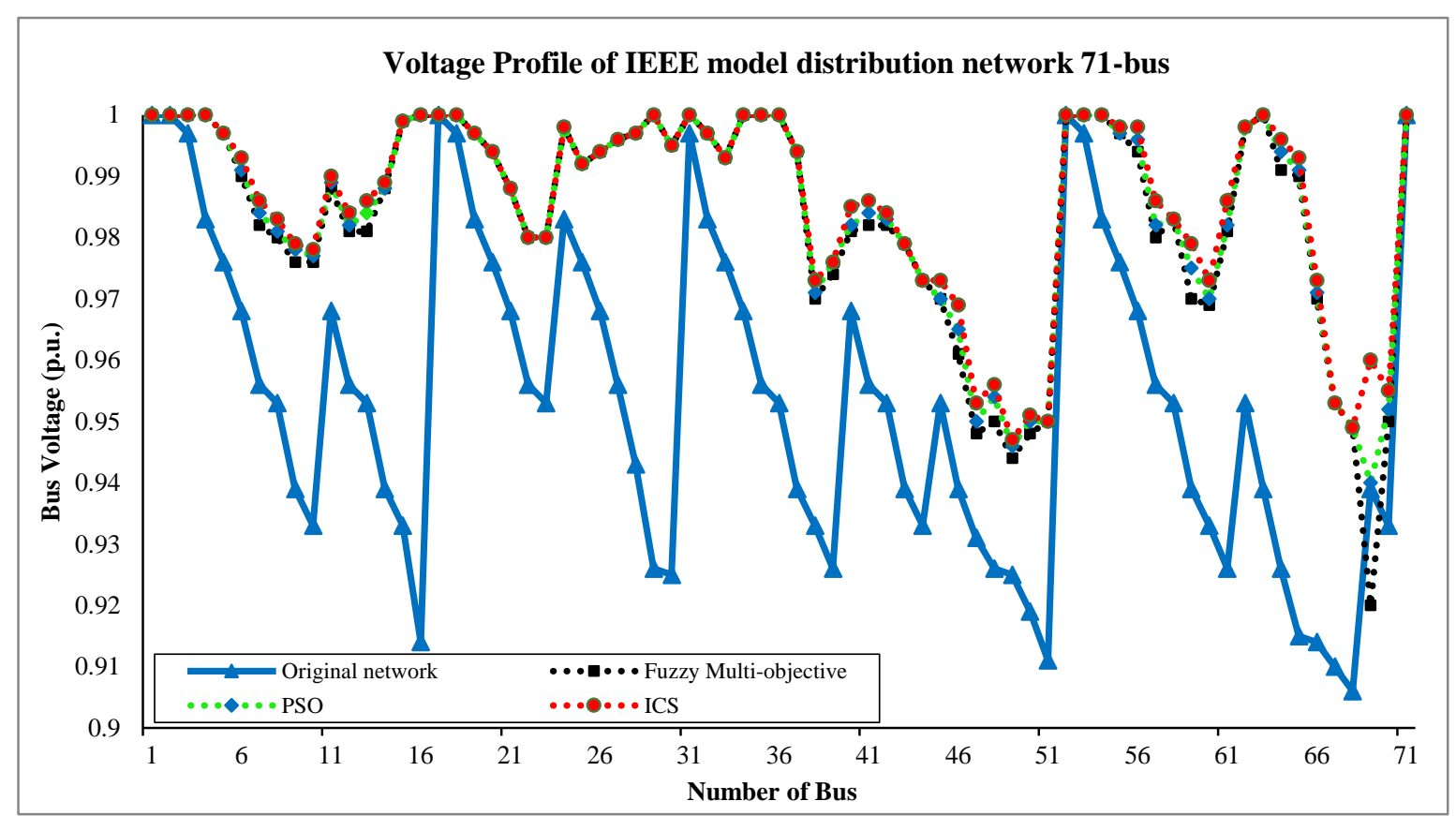

Figure 16. Comparison of voltage profile of the proposed ICS method with fuzzy multi-objective and PSO methods.

In analyzing the performance of the optimization method that has been carried out, it can be reviewed the test data in Table 6. The optimization of the IEEE 71-bus network using the fuzzy multi-objective method produces a power loss of $142.56 \mathrm{~kW}$. In comparison, the PSO method produces $137.23 \mathrm{~kW}$, and the proposed ICS method produces $138.95 \mathrm{~kW}$. The PSO method is superior to the ICS and fuzzy multi-objective methods in reducing power losses. However, if we look at the resulting voltage profile, the ICS method's optimization results are superior to the PSO and fuzzy multiobjective methods. The average bus voltage optimized by the ICS method is 0.986 p.u. While the PSO method produces 0.984 p.u. and the fuzzy multi-objective method yields 0.983 p.u. The voltage profile graphically can be seen in Figure 16. It can be seen that the optimization results using the proposed ICS method make the voltage on most buses have a better magnitude than the PSO and fuzzy multi-objective methods. 
The performance of the ICS method can still be improved by accelerating the cloning and mutation process so that convergence can be achieved in a shorter time. This issue is a part that should be improved in further studies of this research. As seen in the work using the ICS method, the optimization process requires relatively high iterations. The ICS method can be improved in performance by combining other methods such as PSO or fuzzy rules to speed up convergence.

\section{5- Conclusion}

Using the ICS algorithm, the research offered a methodology for multi-objective configuration optimization of the radial distribution network with SG installation. The algorithm can determine the ideal reconfiguration for enhancing the performance of the distribution network being tested. The ICS algorithm was evaluated in this work using an IEEE model distribution network 33-bus and an IEEE model distribution network 71-bus. It was demonstrated numerically that the method effectively decreases the power losses of the two test distribution networks. The ICS method approach has succeeded in improving the performance of the distribution network in the two test systems. In the IEEE 33-bus system test, multi-objective optimization of the network configuration reduced power losses by up to $86.18 \%$. The optimization results also improved the quality of the network voltage from the lowest magnitude of 0.913 to 0.983 p.u., with a maintained load balance on each feeder.

Furthermore, in the IEEE 71-bus system test, multi-objective optimization of the network configuration was also able to reduce power losses by up to $39.13 \%$. The optimization results also improved the quality of the network voltage from the lowest magnitude of 0.906 to 0.948 p.u., with a maintained load balance on each feeder. The improved performance of the IEEE 71-bus network is not as good as that of the IEEE 33-bus network due to its high complexity, namely the number of buses and branches of the IEEE 71-bus network is more than twice as large as that of the IEEE 33-bus network.

\section{6- Nomenclature}

\begin{tabular}{|c|c|c|c|}
\hline$\alpha$ & Factor of cloning or mutation scale, $0<\alpha<1$ & $A b$ & Antibody supplied \\
\hline $\mathrm{d}$ & Antibodies with the lowest affinity that are replaced & $\mathrm{f}_{1}$ & Fitness for the $i$-th random value \\
\hline $\mathrm{f}_{\max }$ & Fitness at its peak & gen & The generational count \\
\hline$I_{i, \min }$ & Lower current magnitude limits at the bus $i$-th. & $I_{i, \max }$ & Upper current magnitude limits at the bus $i$-th. \\
\hline$L$ & String length of each antibody's bits & $N$ & The quantity of antibodies chosen for cloning \\
\hline$n_{b}$ & Branch count & $N_{c}$ & $\begin{array}{l}\text { The clone factor that specifies the scaling factor for the number } \\
\text { of antigen clones }\end{array}$ \\
\hline$P_{\text {loss }}$ & Active power loss has a cost function & $P_{i}$ & Active power exiting of bus $i$-th \\
\hline$Q_{i}$ & Reactive power exiting of bus $i$-th & $Q_{D G}$ & SG's reactive power \\
\hline$R_{i}$ & Resistance at bus $i$-th. & round () & Operators that rotate the input to the closest integer \\
\hline$V_{i}$ & Magnitude of the bus $i$-th voltage & $V_{i, \min }$ & Lower voltage magnitude limits at the bus $i-t h$. \\
\hline$V_{i, \max }$ & Upper voltage magnitude limits at the bus $i$-th. & $\mathrm{X}_{\mathrm{i}+\mathrm{mj}}$ & Parent became mutated (offspring) \\
\hline $\mathrm{X}_{\mathrm{y}}$ & Parents & $\mathrm{X}_{\mathrm{jmax}}$ & $\begin{array}{l}\text { The maximum random number that can be generated for each } \\
\text { variable }\end{array}$ \\
\hline $\mathrm{X}_{\mathrm{jmin}}$ & $\begin{array}{l}\text { The minimum random number that can be generated for } \\
\text { each variable }\end{array}$ & & \\
\hline
\end{tabular}

\section{7- Declarations}

\section{7-1- Author Contributions}

Conceptualization, R.S. and I.S.; methodology, R.S.; software, I.S.; validation, R.S. and I.S.; formal analysis, R.S.; investigation, I.S.; resources, R.S.; data curation, I.S.; writing — original draft preparation, R.S.; writing —review and editing, I.S.; visualization, I.S.; supervision, R.S.; project administration, I.S.; funding acquisition, R.S. All authors have read and agreed to the published version of the manuscript.

\section{7-2-Data Availability Statement}

The data presented in this study are available in article.

\section{7-3- Funding and Acknowledgements}

The authors would like to express their gratitude to Universitas Muhammadiyah Yogyakarta for supporting this work. 


\section{7-4-Conflicts of Interest}

The authors declare that there is no conflict of interests regarding the publication of this manuscript. In addition, the ethical issues, including plagiarism, informed consent, misconduct, data fabrication and/or falsification, double publication and/or submission, and redundancies have been completely observed by the authors.

\section{8- References}

[1] Wang, Jie, Weiqing Wang, Haiyun Wang, and Huiwen Zuo. "Dynamic Reconfiguration of Multiobjective Distribution Networks Considering DG and EVs Based on a Novel LDBAS Algorithm." IEEE Access 8 (2020): 216873-93. doi:10.1109/ACCESS.2020.3041398.

[2] Ali Ehsan, Ming Cheng, and Qiang Yang. "Scenario-Based Planning of Active Distribution Systems under Uncertainties of Renewable Generation and Electricity Demand.” CSEE Journal of Power and Energy Systems 5, no. 1 (2019): 56-62. doi:10.17775/cseejpes.2018.00460.

[3] Song, Yue, Yu Zheng, Tao Liu, Shunbo Lei, and David J. Hill. "A New Formulation of Distribution Network Reconfiguration for Reducing the Voltage Volatility Induced by Distributed Generation.” IEEE Transactions on Power Systems 35, no. 1 (2020): 496-507. doi:10.1109/TPWRS.2019.2926317.

[4] Kianmehr, Ehsan, Saman Nikkhah, Vahid Vahidinasab, Damian Giaouris, and Philip C. Taylor. "A Resilience-Based Architecture for Joint Distributed Energy Resources Allocation and Hourly Network Reconfiguration.” IEEE Transactions on Industrial Informatics 15, no. 10 (2019): 5444-55. doi:10.1109/TII.2019.2901538.

[5] Ahmed, Jubaer, and Zainal Salam. "An Enhanced Adaptive P\&O MPPT for Fast and Efficient Tracking under Varying Environmental Conditions.” IEEE Transactions on Sustainable Energy 9, no. 3 (2018): 1487-96. doi:10.1109/TSTE.2018.2791968.

[6] Sher, Hadeed Ahmed, Khaled E. Addoweesh, and Kamal Al-Haddad. "An Efficient and Cost-Effective Hybrid MPPT Method for a Photovoltaic Flyback Microinverter." IEEE Transactions on Sustainable Energy 9, no. 3 (2018): 1137-44. doi:10.1109/TSTE.2017.2771439.

[7] Liu, Zifa, Yunyang Liu, Gaoqiang Qu, Xinyue Wang, and Xuyang Wang. “Intra-Day Dynamic Network Reconfiguration Based on Probability Analysis Considering the Deployment of Remote Control Switches.” IEEE Access 7 (2019): 145272-81. doi:10.1109/ACCESS.2019.2944917.

[8] Syahputra, Ramadoni, and Indah Soesanti. "Planning of Hybrid Micro-Hydro and Solar Photovoltaic Systems for Rural Areas of Central Java, Indonesia.” Journal of Electrical and Computer Engineering 2020 (2020). doi:10.1155/2020/5972342.

[9] Syahputra, Ramadoni, and Indah Soesanti. "Performance Improvement for Small-Scale Wind Turbine System Based on Maximum Power Point Tracking Control.” Energies 12, no. 20 (2019): 3938. doi:10.3390/en12203938.

[10] Al-Ismail, Fahad S. "Discussion on 'A New Formulation of Distribution Network Reconfiguration for Reducing the Voltage Volatility Induced by Distributed Generation.” IEEE Transactions on Power Systems 35, no. 6 (2020): 4974. doi:10.1109/TPWRS.2020.3012366.

[11] Syahputra, Ramadoni, and Indah Soesanti. "Renewable Energy Systems Based on Micro-Hydro and Solar Photovoltaic for Rural Areas: A Case Study in Yogyakarta, Indonesia.” Energy Reports 7 (2021): 472-90. doi:10.1016/j.egyr.2021.01.015.

[12] Hien, Nguyen Cong, Nadarajah Mithulananthan, and R. C. Bansal. "Location and Sizing of Distributed Generation Units for Loadabilty Enhancement in Primary Feeder.” IEEE Systems Journal 7, no. 4 (2013): 797-806. doi:10.1109/JSYST.2012.2234396.

[13] Azizivahed, Ali, Ali Arefi, Sahand Ghavidel, Miadreza Shafie-Khah, Li Li, Jiangfeng Zhang, and Joao P.S. Catalao. "Energy Management Strategy in Dynamic Distribution Network Reconfiguration Considering Renewable Energy Resources and Storage.” IEEE Transactions on Sustainable Energy 11, no. 2 (2020): 662-73. doi:10.1109/TSTE.2019.2901429.

[14] Lei, Shunbo, Chen Chen, Yue Song, and Yunhe Hou. "Radiality Constraints for Resilient Reconfiguration of Distribution Systems: Formulation and Application to Microgrid Formation.” IEEE Transactions on Smart Grid 11, no. 5 (2020): 3944-56. doi:10.1109/TSG.2020.2985087.

[15] Sekhavatmanesh, Hossein, and Rachid Cherkaoui. “A Multi-Step Reconfiguration Model for Active Distribution Network Restoration Integrating DG Start-Up Sequences.” IEEE Transactions on Sustainable Energy 11, no. 4 (2020): $2879-88$. doi:10.1109/TSTE.2020.2980890.

[16] Calderaro, V., A. Piccolo, and P. Siano. "Maximizing DG Penetration in Distribution Networks by Means of GA Based Reconfiguration." In 2005 International Conference on Future Power Systems, Vol. 2005. Amsterdam, 2005. doi:10.1109/fps.2005.204237. 
[17] Wang, Ying, Yin Xu, Jiaxu Li, Jinghan He, and Xiaojun Wang. "On the Radiality Constraints for Distribution System Restoration and Reconfiguration Problems." IEEE Transactions on Power Systems 35, no. 4 (2020): 3294-96. doi:10.1109/TPWRS.2020.2991356.

[18] Huang, Wanjun, Weiye Zheng, and David J. Hill. "Distributionally Robust Optimal Power Flow in Multi-Microgrids with Decomposition and Guaranteed Convergence." IEEE Transactions on Smart Grid 12, no. 1 (2021): 43-55. doi:10.1109/TSG.2020.3012025.

[19] Akrami, Alireza, Meysam Doostizadeh, and Farrokh Aminifar. "Optimal Reconfiguration of Distribution Network Using Imu PMU Measurements: A Data-Driven Stochastic Robust Optimization.” IEEE Transactions on Smart Grid 11, no. 1 (2020): 42028. doi:10.1109/TSG.2019.2923740.

[20] Zheng, Weiye, Wanjun Huang, David J. Hill, and Yunhe Hou. “An Adaptive Distributionally Robust Model for Three-Phase Distribution Network Reconfiguration.” IEEE Transactions on Smart Grid 12, no. 2 (2021): $1224-37$. doi:10.1109/TSG.2020.3030299.

[21] Ahmed, Haytham M.A., and Magdy M.A. Salama. "Energy Management of AC-DC Hybrid Distribution Systems Considering Network Reconfiguration." IEEE Transactions on Power Systems 34, no. $6 \quad$ (2019): 4583-94. doi:10.1109/TPWRS.2019.2916227.

[22] Millar, R. J., E. Saarijärvi, M. Lehtonen, M. Hyvärinen, J. Niskanen, and P. Hämäläinen. "Electricity Distribution Network Planning Algorithm Based on Efficient Initial and Radial-to-Full Network Conversion.” International Review of Electrical Engineering 8, no. 3 (2013): 1076-90. doi:10.15866/iree.v8i3.1870.

[23] Farahani, Vahid, Behrooz Vahidi, and Hossein Askarian Abyaneh. "Reconfiguration and Capacitor Placement Simultaneously for Energy Loss Reduction Based on an Improved Reconfiguration Method.” IEEE Transactions on Power Systems 27, no. 2 (2012): 587-95. doi:10.1109/TPWRS.2011.2167688.

[24] Das, Debapriya. “A Fuzzy Multiobjective Approach for Network Reconfiguration of Distribution Systems.” IEEE Transactions on Power Delivery 21, no. 1 (2006): 202-9. doi:10.1109/TPWRD.2005.852335.

[25] Syahputra, Ramadoni, Imam Robandi, and Mochamad Ashari. "Optimization of Distribution Network Configuration with Integration of Distributed Energy Resources Using Extended Fuzzy Multi-Objective Method.” International Review of Electrical Engineering 9, no. 3 (2014): 629-39. doi:10.15866/iree.v9i3.956.

[26] Jeon, Young Jae, Jae Chul Kim, Jin O. Kim, Joong Rin Shin, and Kwang Y. Lee. “An Efficient Simulated Annealing Algorithm for Network Reconfiguration in Large-Scale Distribution Systems.” IEEE Transactions on Power Delivery 17, no. 4 (2002): 1070-78. doi:10.1109/TPWRD.2002.803823.

[27] Enacheanu, Bogdan, Bertrand Raison, Raphael Caire, Olivier Devaux, Wojciech Bienia, and Nouredine HadjSaid. "Radial Network Reconfiguration Using Genetic Algorithm Based on the Matroid Theory." IEEE Transactions on Power Systems 23, no. 1 (2008): 186-95. doi:10.1109/TPWRS.2007.913303.

[28] Srinivasa Rao, Rayapudi, Sadhu Venkata Lakshmi Narasimham, Manyala Ramalinga Raju, and A. Srinivasa Rao. "Optimal Network Reconfiguration of Large-Scale Distribution System Using Harmony Search Algorithm.” IEEE Transactions on Power Systems 26, no. 3 (2011): 1080-88. doi:10.1109/TPWRS.2010.2076839.

[29] Falaghi, Hamid, Mahmood Reza Haghifam, and Chanan Singh. "Ant Colony Optimization-Based Method for Placement of Sectionalizing Switches in Distribution Networks Using a Fuzzy Multiobjective Approach.” IEEE Transactions on Power Delivery 24, no. 1 (2009): 268-76. doi:10.1109/TPWRD.2008.2005656.

[30] Niknam, Taher, Hamed Zeinoddini Meymand, and Hasan Doagou Mojarrad. "A Practical Multi-Objective PSO Algorithm for Optimal Operation Management of Distribution Network with Regard to Fuel Cell Power Plants.” Renewable Energy 36, no. 5 (2011): 1529-44. doi:10.1016/j.renene.2010.11.027.

[31] Syahputra, Ramadoni, Imam Robandi, and Mochamad Ashari. "Performance Improvement of Radial Distribution Network with Distributed Generation Integration Using Extended Particle Swarm Optimization Algorithm.” International Review of Electrical Engineering 10, no. 2 (2015): 293-304. doi:10.15866/iree.v10i2.5410.

[32] Syahputra, Ramadoni, Indah Soesanti, and Mochamad Ashari. "Performance Enhancement of Distribution Network with Dg Integration Using Modified PSO Algorithm.” Journal of Electrical Systems 12, no. 1 (2016): 1-19.

[33] Ahmad, N. H., T. K.A. Rahman, and N. Aminuddin. "Multi-Objective Quantum-Inspired Artificial Immune System Approach for Optimal Network Reconfiguration in Distribution System.” In 2012 IEEE International Power Engineering and Optimization Conference, PEOCO 2012 - Conference Proceedings, 384-88. Melaka, Malaysia, 2012. doi:10.1109/PEOCO.2012.6230894.

[34] Chen, Qian, Weiqing Wang, Haiyun Wang, Jiahui Wu, Xiaozhu Li, and Jiongfeng Lan. “A Social Beetle Swarm Algorithm Based on Grey Target Decision-Making for a Multiobjective Distribution Network Reconfiguration Considering Partition of Time Intervals.” IEEE Access 8 (2020): 204987-13. doi:10.1109/ACCESS.2020.3036898. 
[35] Hu, Tianyu, Qinglai Guo, Zhengshuo Li, Xinwei Shen, and Hongbin Sun. "Distribution-Free Probability Density Forecast through Deep Neural Networks.” IEEE Transactions on Neural Networks and Learning Systems 31, no. 2 (2020): 612-25. doi:10.1109/TNNLS.2019.2907305.

[36] Zhong, Tianwei, Hai Tao Zhang, Yuanzheng Li, Lan Liu, and Renzhi Lu. "Bayesian Learning-Based Multi-Objective Distribution Power Network Reconfiguration.” IEEE Transactions on Smart Grid 12, no. 2 (2021): 1174-84. doi:10.1109/TSG.2020.3027290.

[37] Wang, Wei, Nanpeng Yu, Yuanqi Gao, and Jie Shi. "Safe Off-Policy Deep Reinforcement Learning Algorithm for Volt-VAR Control in Power Distribution Systems." IEEE Transactions on Smart Grid 11, no. 4 (2020): 3008-18. doi:10.1109/TSG.2019.2962625.

[38] Zheng, Weiye, Wanjun Huang, and David J. Hill. “A Deep Learning-Based General Robust Method for Network Reconfiguration in Three-Phase Unbalanced Active Distribution Networks.” International Journal of Electrical Power and Energy Systems 120 (2020). doi:10.1016/j.ijepes.2020.105982.

[39] Gao, Yuanqi, Wei Wang, Jie Shi, and Nanpeng Yu. "Batch-Constrained Reinforcement Learning for Dynamic Distribution Network Reconfiguration.” IEEE Transactions on Smart Grid 11, no. 6 (2020): 5357-69. doi:10.1109/TSG.2020.3005270.

[40] Li, Zihao, Wenchuan Wu, Boming Zhang, and Xue Tai. “Analytical Reliability Assessment Method for Complex Distribution Networks Considering Post-Fault Network Reconfiguration.” IEEE Transactions on Power Systems 35, no. 2 (2020): $1457-67$. doi:10.1109/TPWRS.2019.2936543.

[41] Phu-Ang, Ajchara. “An Improve Artificial Immune Algorithm for Solving the Travelling Salesman Problem.” In 2021 Joint 6th International Conference on Digital Arts, Media and Technology with 4th ECTI Northern Section Conference on Electrical, Electronics, Computer and Telecommunication Engineering, ECTI DAMT and NCON 2021, 261-64, 2021. doi:10.1109/ECTIDAMTNCON51128.2021.9425773.

[42] Zhang, Liangpei, Yanfei Zhong, and Pingxiang Li. "Applications of Artificial Immune Sysetms in Remote Sensing Image Classification.” In Proc. 20th Congress Int. Soc. for Photogrammetry and Remote Sensing, Vol. 35, 35:397-401. Sens., Istanbul, Turkey, (2004). 